\title{
Hadronic vacuum-polarization contribution to various QED observables
}

\author{
Savely G. Karshenboim ${ }^{1,2,3, a}$ and Valery A. Shelyuto ${ }^{4}$ \\ 1 Ludwig-Maximilians-Universität, Fakultät für Physik, 80799 München, Germany \\ 2 Max-Planck-Institut für Quantenoptik, 85748 Garching, Germany \\ 3 Pulkovo Observatory, St.Petersburg, Russia 196140 \\ ${ }^{4}$ D. I. Mendeleev Institute for Metrology, St.Petersburg, Russia 190005
}

Received 14 September 2020 / Accepted 15 January 2021 / Published online 8 February 2021 (C) The Author(s) 2021

\begin{abstract}
Due to precision tests of quantum electrodynamics (QED), determination of accurate values of fundamental constants, and constraints on new physics, it is important in a consistent way to evaluate a number of QED observables such as the Lamb shift in hydrogen-like atomic systems. Even in a pure leptonic case, those QED variables are in fact not pure QED ones since hadronic effects are involved through intermediate states while accounting for higher-order effects. One of them is hadronic vacuum polarization (hVP). Complex evaluations often involve a number of QED quantities, for which treatment of $h V P$ is not consistent. The highest accuracy for a calculation of the hVP term is required for the anomalous magnetic moment of a muon. However, a standard data-driven treatment of hVP, based on a dispersion integration of experimental data on electron-positron annihilation to hadrons and some other phenomena, leads to a contradiction with the experimental value of $a_{\mu}$. This experimental value can be considered as an indirect determination of the hVP contribution to $a_{\mu}$ and the scatter of theory and experiment allows one to obtain a conservative estimation of the related $\mathrm{hVP}$ contribution. In this paper, we derive exact and approximate relations between the leading-order (LO) hVP contributions to various observables. Using those relations, we obtain for them a consistent set of the results, based on the scatter of $a_{\mu}$ values. While calculating the LO hVP term, we have to remember that next-to-LO (NLO) hVP corrections are often comparable with the uncertainty of the LO term. Special attention is payed to hVP contribution to simple atoms. In particular, we discuss the NLO contribution to the Lamb shift in ordinary and muonic hydrogen and other two-body atoms for $Z \leq 10$. We also consider the NLO contribution of the muonic vacuum polarization to the Lamb shift in hydrogen-like atoms. With the $a_{\mu}$ puzzle unresolved, one may still require present-days values of the $\mathrm{hVP}$ contributions to various observable for comparison to experiment etc. the presence of contradicting values and a lack of consistency means an additional uncertainty for $a_{\mu}$ and for key contributions to it, including the LO hVP one. We present here an estimation of such a propagated uncertainty in hVP contributions to different QED observables and recommend a consistent set of the related LO hVP contributions.
\end{abstract}

\section{Introduction}

Precision low-energy tests of quantum electrodynamics are not entirely pure QED tests. Even in the case of purely leptonic systems, theory requires a certain hadronic input and, in particular, an input from hadronic vacuum polarization (hVP).

There is a number of QED observables for which we need such hVP contributions. The most important one is the anomalous magnetic moment of a muon $a_{\mu}$. A calculation of the leading hVP contribution to $a_{\mu}$ at the level better than one percent is required for a comparison of theory and experiment (see, e.g., [1]). Other observables include the anomalous magnetic moment of an electron $a_{e}$, muonium hyperfine (Mu HFS) inter-

\footnotetext{
${ }^{a}$ e-mail: savely.karshenboim@mpq.mpg.de (corresponding author)
}

val $\nu_{\mathrm{Mu}}$ (of the $1 s$ state), the Lamb shift in hydrogen $E_{L}(\mathrm{H})$ for various states, the most important of which is $1 s$, and the Lamb shift in muonic hydrogen $E_{L}(\mu \mathrm{H})$ for the $2 s$ state or, more accurately for the $2 p-2 s$ interval. Contributions to the Lamb shift in other ordinary and muonic light atoms such as $[\mu] \mathrm{D},[\mu]^{3} \mathrm{He}^{+}$, and $[\mu]^{4} \mathrm{He}^{+}$are also required for theoretical evaluation and interpretation of experimental data.

While the leading-order (LO) term for $\Delta a_{\mu}(\mathrm{hVP})$ has been recently accurately calculated in many papers, the LO contributions for $\Delta a_{e}(\mathrm{hVP})([2,3])$ and for $\Delta \nu_{\mathrm{Mu}}(\mathrm{hVP})([2])$ are found in a few publications only. There are also not very accurate calculations of the related contributions to Lamb shift in $\mathrm{H}[4]$ and $\mu \mathrm{H}$ (see, e.g., [5]). A number of results on the next-to-leadingorder (NLO) hVP contributions to $a_{\mu}$ and LO and NLO 
hVP contributions to some other observables, which do not require a high accuracy, exist as well.

Even if the required accuracy of the other applications is not as high as that for $a_{\mu}$, there is still a question of a consistent evaluation of all the hVP contributions once one makes a 'grand QED evaluation' with many observables. Such an evaluation would be helpful for a determination of values of fundamental constants (see, e.g., [6]) or for a 'gross QED test' to constrain new physics.

Here we consider relations between the leading hVP contributions to various QED observables. Some of the relations are exact, others are approximate. All the corrections can be presented in a generic form, since they are proportional to an integral

$$
\mathcal{I}=\int \mathrm{d} s \rho(s) K(s)
$$

over a dispersion variable $s$. Here $K(s)$ is a result of an integration over a QED part of the diagrams, usually expressed in terms of elementary functions, and

$$
\rho(s)=\frac{R(s)}{3 s}
$$

is a quantity experimentally determined from $e^{+} e^{-}$annihilation to hadrons or extracted from similar phenomena. Accurate calculations may require interpolations or extrapolations of the existing data as well as theoretical partly model-dependent input in the area where the data are not very accurate or not available at all. Until recently the dispersion integration in (1) was considered as the only way to find LO hVP contributions, however recently lattice calculations started delivering results with comparable accuracy.

The purpose of our work here is not to find an hVP contribution by itself, say, to Mu HFS interval from scratch, but to check how a change in $R(s)$, due to a possible update of the experimental data or corrections, could affect the existing results. We take advantage of exact and approximate relations between $K$ 's for various observables which we derive in this paper. Such approximate relations may be not sufficient to find a contribution by itself, however for a small correction (compared to a previously obtained value) the approximate relations should be sufficient. They can be also used to consider the uncertainty due to a scatter of the data and discrepancies. One more application of the approximate relations is due to higher-order hVP contributions, for which the high accuracy is not required.

The variety of possible results for the $R$ function is not entirely due to its successful improvement. There is a scatter of the experimental results obtained in different experiments and/or evaluated by different methods. Therefore, different existing parameterizations of the $R$ function could be in part inconsistent.

There are two very different groups of problems in hVP calculations with the $R$ function for various QED observables.
- For pure leptonic cases $\left(a_{\mu}, a_{e}\right.$, and $\left.\nu_{\mathrm{Mu}}\right)$, the hVP contribution should be taken into account. The required fractional accuracy for the leading hVP term differs from an application to application. The highest one is for $\Delta a_{\mu}(\mathrm{hVP})$. One of the problems is an internal theoretical problem - the $R$ function, determined from different groups of data or with help of corrections being approached differently, has inconsistencies. That should increase the uncertainty. The spread is present in the calculation of $\Delta a_{\mu}(\mathrm{hVP})$ (see, e.g., [1]), but not for two other leptonic observables. For them the uncertainty due to the scatter of the results should be estimated.

A bigger problem is a discrepancy between theory and experiment for $a_{\mu}$. There are four options to resolve the discrepancy. One is 'new physics'. Once a model of new physics is introduced to force agreement between theory and experiment for $a_{\mu}$, it should be considered for the other observables. The second option is a possible problem with the experiment on $a_{\mu}$ [7]. The third one is due to a possible incorrectness of the hVP calculations (questions about the required [raw] data for $R(s)$ and corrections for measurements, say, radiative corrections). The fourth option is a problem with a contribution of hadronic light-by-light scattering (hLbL). Other possibilities are unlikely. For all the options without involvement of new physics, we can consider either corrections (one can correct various values to reach agreement or choose which of the values is a 'correct' one) or an increase in the related uncertainties. Note, in the case of Mu HFS interval we need not only $\Delta \nu_{\mathrm{Mu}}(\mathrm{hVP})$, but also a value of $a_{\mu}$ to calculate the leading term, and a certain hLBL correction to find one of the higher-order hadronic contributions. Their treatment should be consistent with the interpretation of the $a_{\mu}$ discrepancy.

Let us consider one of the options - the $a_{\mu}$ experiment is correct, while the hLbL contribution is reasonably estimated. Then, we can extract

$$
\Delta a_{\mu}^{\text {from expt }}(\mathrm{LO} \mathrm{hVP})=720.1(6.9) \times 10^{-10}
$$

from a comparison of theory (without the leading hVP term) [3] and experiment. Indeed, such an interpretation would affect $\Delta a_{e}(\mathrm{LO} \mathrm{hVP})$ and $\Delta \nu_{\mathrm{Mu}}(\mathrm{LO} \mathrm{hVP})$, probably increasing their values by a few percent. This example demonstrates the importance of the interpretation of the $a_{\mu}$ discrepancy for other observables. The shift is definitely a few times larger than the uncertainty of any 'direct' theoretical calculation for $\Delta a_{e}(\mathrm{LO} h V \mathrm{P})$, $\Delta \nu_{\mathrm{Mu}}(\mathrm{LO}$ hVP) etc.

Another alternative is to consider the scatter in determination of $a_{\mu}$ (i.e., the very difference in experimental and theoretical values) as an estimation of the uncertainty in determination of 
$\Delta a_{\mu}(\mathrm{LO} \mathrm{hVP})$. In this case, we have to define the latter as

$$
\Delta a_{\mu}^{\text {from scat }}(\mathrm{LO} \mathrm{hVP})=705(15) \times 10^{-10},
$$

which would likely increase the other hVP values by a few percent (but less that for the related results 'from experiment') and set their uncertainty to a level of a few percent (essentially higher that for the results 'from experiment').

Use of either of those two values of $\Delta a_{\mu}(\mathrm{hVP})$ for other observables should push the related hVP contributions for other observables beyond the present uncertainty of their direct calculations. A correct interpretation of any hVP contribution requires establishment of a relation between $\Delta a_{\mu}(\mathrm{hVP})$ and the hVP contribution to an observable quantity of interest.

- Observables with hadronic nuclei, such as the Lamb shift in ordinary and muonic atoms, should be treated differently. We cannot measure the nuclear radii, but we can extract their value from the electric-charge form factors. Meanwhile, we basically measure not the electric form factor $G_{E}\left(q^{2}\right)$, but a value $G_{E}\left(q^{2}\right) /\left(1-\pi_{h}\left(q^{2}\right)\right)$ (see, e.g., a discussion in [4]). Here $\pi_{h}\left(q^{2}\right)$ is a certain factor related to hadronic vacuum polarization. That means that any 'incorrect' treatment of the hadronic VP means rather an effective redefinition of the electric form factor and the related rms charge radius. At the present level of accuracy, such a 'redefinition' of the charge radius affects neither a determination of $R_{\infty}$, nor internal consistency of evaluation of data related to $\mathrm{H}, \mu \mathrm{H}, \mathrm{D}, \mu \mathrm{D}$, etc., as far as the hVP factor $\left(1-\pi_{h}\left(q^{2}\right)\right)$ is introduced in all the observables in a consistent way and as far as all the related nuclei are characterized by the data on the elastic electronnucleus scattering. In such a case, the hVP contributions can be completely ignored and that would not lead to any problem in the interpretation.

However, the situation with compound nuclei is not that simple as for the hydrogen nucleus, a proton. For a proton, one has no alternative to the scattering data and the measured quantity is expressed through $G_{E}\left(q^{2}\right) /\left(1-\pi_{h}\left(q^{2}\right)\right)$. For heavier nuclei, starting with the deuteron, one can construct an efficient nuclear model. In this case, the model 'speaks' rather directly in terms of $G\left(q^{2}\right)$ and the hVP correction should be added. Often the radius itself is obtained from scattering, while a model describes various higher-order nuclear-structure effects, but still should produce a reasonable value of the radius as a consistency test.

Once the overall set of available data includes both $\mathrm{H}$ and $\mathrm{D}$ atoms and their muonic analogues, a certain consistent treatment of hVP is required.

Numerically, the mentioned effects are of minor

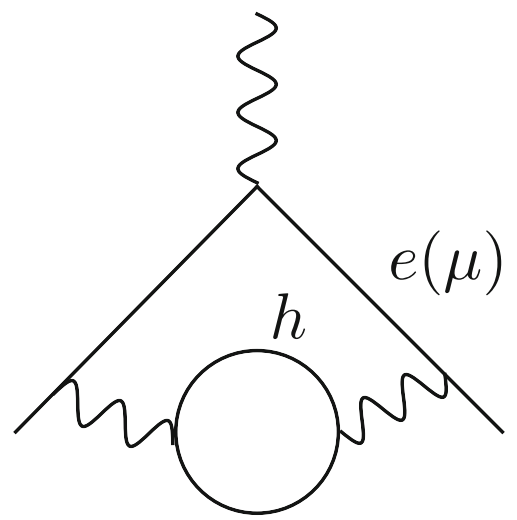

Fig. 1 The LO hVP contribution to the anomalous magnetic moment of an electron (a muon)

importance for an evaluation of the current data; however, their importance may change with increase in the accuracy and increase in the nuclear charge $Z$. It is also important to be in control of those small corrections to be on a safe side.

With a dispersion-integral substitution for the Euclidean momentum $q^{2}$

$$
\frac{1}{q^{2}} \rightarrow \frac{\alpha}{\pi} \int \frac{\mathrm{d} s \rho(s)}{q^{2}+s}
$$

one can present the result for a $\mathrm{LO} \mathrm{hVP}$ contribution of the observable $X_{i}$ as

$$
\Delta X_{i}(\mathrm{hVP})=C_{i} \int \mathrm{d} s \rho(s) K_{i}\left(s, m_{i}\right)
$$

Here, $C_{i}$ is a normalization constant (see Table 3 ) and $m_{i}$ is a characteristic mass for the observable $X_{i}$. For $X$ being the Lamb shift for an $n s$ state in hydrogen (or other H-like atoms) and $a_{e}, m_{i}=m_{e}$, while for $a_{\mu}, \nu_{\mathrm{Mu}}$ and the Lamb shift in $\mu \mathrm{H}$ (and other muonic atoms) $m_{i}=m_{\mu}$. As mentioned, often a parameterization for the dispersion density $\rho(s)$ in terms of the $R$ function [see (2)] is utilized.

The most important property of the expression (5) is that the QED kernels $K_{i}(s)$ are known [8-11] or can be relatively easily derived for the leading $\mathrm{hVP}$ contributions to various QED observables, while the $R$ function can be expressed through a cross section of the $e^{+} e^{-}$ annihilation to hadrons, which is measured (or through similar effects such as the rate of hadronic modes of $\tau$ decay). That makes the outcome for different observables $X_{i}$ correlated. Comparing various $K_{i}$ we are to identify those correlations.

The kernels for the leading-order hVP contributions are known. The result for the anomalous magnetic moment of a muon $\left(m=m_{\mu}\right)$ or an electron $\left(m=m_{e}\right)$ 

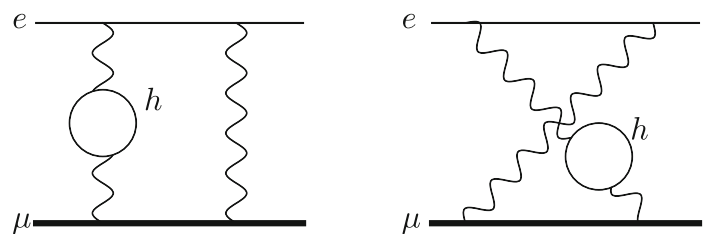

Fig. 2 The leading hadronic contribution to the muonium HFS interval

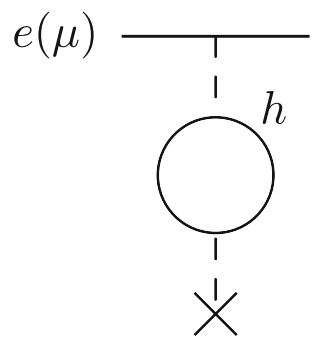

Fig. 3 The LO hVP contribution to the Lamb shift in hydrogen and muonic hydrogen

(see Fig. 1) is of the form [8-10]

$$
\begin{aligned}
K_{\mathrm{a}}(s)= & -\frac{\frac{s^{2}}{2 m^{4}}-\frac{2 s}{m^{2}}+1}{\sqrt{1-\frac{4 m^{2}}{s}}} \ln \frac{1+\sqrt{1-\frac{4 m^{2}}{s}}}{1-\sqrt{1-\frac{4 m^{2}}{s}}} \\
& +\left(\frac{s^{2}}{2 m^{4}}-\frac{s}{m^{2}}\right) \ln \frac{s}{m^{2}}-\frac{s}{m^{2}}+\frac{1}{2} ;
\end{aligned}
$$

the kernel for the LO hVP contribution to the $1 s \mathrm{Mu}$ HFS interval $\left(m=m_{\mu}\right)$ (see Fig. 2) reads [11] (see also [12])

$$
\begin{aligned}
K_{\mathrm{Mu}}(s)= & -\left(\frac{s}{4 m^{2}}+2\right) \sqrt{1-\frac{4 m^{2}}{s}} \ln \frac{1+\sqrt{1-\frac{4 m^{2}}{s}}}{1-\sqrt{1-\frac{4 m^{2}}{s}}} \\
& +\left(\frac{s}{4 m^{2}}+\frac{3}{2}\right) \ln \frac{s}{m^{2}}-\frac{1}{2} ;
\end{aligned}
$$

while the result for the Lamb shift in $\mathrm{H}\left(m=m_{e}\right)$ and $\mu \mathrm{H}\left(m=m_{\mu}\right)$ (see Fig. 3) is obvious

$$
K_{\mathrm{L}}(s)=\frac{m^{2}}{s}
$$

(Here and throughout the paper we apply units in which $\hbar=c=1$.)

Sometimes calculations (within a 'big evaluation') deal with different $R$ functions for different observables $X_{i}$ (see, e.g., [6]). (Indeed, nobody applies by themselves different $R$ 's parameterizations within one paper, but frequently results, which have already been obtained in various original papers with different $R$ 's for different $X$ 's, are utilized within a single review or compilation without any reservations.) Such an evaluation is inconsistent. A consistent one would suggest the use of the same $R$ for all the observables involved.

We intend to find the corrections to calculation of various $\mathrm{hVP}$ contributions. One of the purposes is to be able to correct and to make consistent the results for different $X$ 's for the case when the original evaluation has been performed with different $R$ 's. To do that we should be able to find what would happen with values of the hVP contributions to two observables if we [slightly] change the $R$ function.

Having $R(s)$, it is possible to find the LO hVP contribution to any observable directly. We intend to consider relations between the LO hVP contributions for two reasons. Indeed, the relations will simplify an adaptation of a result of a calculation on one observable to another. (We remind that there is quite a large number of different evaluations of $\mathrm{LO} \mathrm{hVP}$ for $a_{\mu}$ and a relatively few calculations for other observables. We prepare an 'instrument' to adjust any result for $a_{\mu}$ to a calculation for another observable.) As we mentioned, a direct calculation is possible for all observables and such relations would only simplify the adaptation of direct results for $a_{\mu}(\mathrm{LO}$ hVP) to other quantities. In the meantime, we also have to deal with indirect results on $a_{\mu}(\mathrm{LO} \mathrm{hVP})$ which follow from a comparison of theory and experiment (see Eqs. 3 and 4)). Those indirect results are not related to any set of data on $R$ (or related only in part). In this case, we need relations to convert a result on $a_{\mu}$ to other observables. Similar situation exists with adaptation of lattice results $a_{\mu}(\mathrm{LO} \mathrm{hVP})$. Those are direct results, but they are not based on a dispersion presentation (6).

With the so-called $a_{\mu}$ puzzle still unresolved, there is no consensus choice of a value of $a_{\mu}$. It is uncertain due to the discrepancy and the uncertainty is essentially larger than [claimed] uncertainties of individual theoretical or experimental determinations. Since we have no preferences on the resolution of the discrepancy, such an uncertainty should be considered as an uncertainty of all the involved key contributions including the one due to hVP. Once we consider $\Delta a_{\mu}(\mathrm{hVP})$ more uncertain than its individual determinations [(cf. (4)], we have to acknowledge that until a consensus on the solution of the $a_{\mu}$ puzzle is reached the experimental part of the dispersion integrand for $\Delta a_{\mu}(\mathrm{hVP})$ [(cf. (6)] is compromised and an additional uncertainty should be assigned to it. That in its turn means an additional uncertainty for all calculations with $R(s)$. We demonstrate below that certain relations between hVP contributions to different QED observables could serve this purpose. We stress that we consider an error in $R(s)$ just as a possible solution of the problem on the same ground as the other possibilities (an experimental error in $a_{\mu}$, a theoretical error in hLbL, or new physics). 
Table 1 LO hVP contribution to various QED observables. Here, $\widetilde{E}_{F}=8 / 3(Z \alpha)^{4} m_{r}^{3} /\left(m_{e} m_{\mu}\right)$ is the leading nonrelativistic (NR) HFS term, which includes only the Dirac's values of the lepton magnetic moments (see Sect. 4); $m_{r}$ is the reduced mass of the related two-body system (muonium $e \mu$, hydrogen $e p$, muonic hydrogen $\mu p$ ). $Z$ is the nuclear charge which is equal to unity for all the mentioned two-body atoms; however, the result may be generalized to other hydrogen-like atoms with $Z \neq 1$ as we do for the Lamb shift in this paper

\begin{tabular}{llll}
\hline$X_{i}$ & $C_{i}$ & $K_{i}\left(m_{i}, s\right)$ & $m_{i}$ \\
\hline$a_{\mu}$ & $(\alpha / \pi)^{2}$ & Eq. $(7)$ & $m_{\mu}$ \\
$a_{e}$ & $(\alpha / \pi)^{2}$ & $1 / 3\left(m_{i}^{2} / s\right)$ & $m_{e}$ \\
$\Delta E_{L}(1 s, \mathrm{H})$ & $-4(\alpha / \pi)(Z \alpha)^{4} m_{r}^{3} / m_{e}^{2}$ & $\left(m_{i}^{2} / s\right)$ & $m_{e}$ \\
$\Delta E_{L}(2 s, \mu \mathrm{H})$ & $-(\alpha / 2 \pi)(Z \alpha)^{4} m_{r}^{3} / m_{\mu}^{2}$ & $\left(m_{i}^{2} / s\right)$ & $m_{\mu}$ \\
$\nu_{\mathrm{Mu}}$ & $2(\alpha / \pi)^{2}\left(m_{e} / m_{\mu}\right) \widetilde{E}_{F}$ & Eq. (8) & $m_{\mu}$ \\
\hline
\end{tabular}

Table 2 LO hVP contributions to $a_{e}$ and Lamb shift in hydrogen and muonic hydrogen. The average has the same uncertainty as individual contributions since both original results are based on experimental data, a large portion of which is the same

\begin{tabular}{lllll}
\hline Ref. & $\Delta a_{e}\left[10^{-12}\right]$ & $m_{e}^{2} \Pi_{h}^{\prime}(0)\left[10^{-6}\right]$ & $\Delta E_{L}(1 s)$ in $\mathrm{H}[\mathrm{Hz}]$ & $\Delta E(2 p-2 s)$ in $\mu \mathrm{H}[\mathrm{meV}]$ \\
\hline$[2]$ & $1.866(11)$ & $1.038(6)$ & $-3372(20)$ & $0.01121(7)$ \\
{$[3]$} & $1.849(11)$ & $1.028(6)$ & $-3341(20)$ & $0.01111(7)$ \\
\hline Average & $1.858(11)$ & $1.033(6)$ & $-3357(20)$ & $0.01116(7)$ \\
\hline
\end{tabular}

\section{Slope of the hadronic vacuum polarization $\Pi_{h}^{\prime}(0)$ and LO hVP contributions to various observables}

As seen from Table 1, some kernels are of a simple form and in particular a few of them are $\propto \mathrm{m}^{2} / \mathrm{s}$. Let us introduce a notation for the slope of hVP polarization at zero

$$
\Pi_{h}^{\prime}(0)=\int \frac{\mathrm{d} s \rho(s)}{s} .
$$

The slope is given for the vacuum polarization as a function of the Euclidean momentum. The slope for the polarization as a function of the standard Minkovsky four-momentum has the opposite sign.

In the terms of the slope, we find

$$
\begin{aligned}
\Delta a_{e}(\mathrm{LO} \mathrm{hVP}) & =\frac{1}{3}\left(\frac{\alpha}{\pi}\right)^{2} m_{e}^{2} \Pi_{h}^{\prime}(0), \\
\Delta E_{L}(\mathrm{LO} \mathrm{hVP}, 1 s, \mathrm{H}) & =-\frac{4 \alpha(Z \alpha)^{4} m_{r}^{3}}{\pi} \Pi_{h}^{\prime}(0), \\
\Delta E_{L}(\mathrm{LO} \mathrm{hVP}, 2 s, \mu \mathrm{H}) & =-\frac{\alpha(Z \alpha)^{4} m_{r}^{3}}{2 \pi} \Pi_{h}^{\prime}(0) .
\end{aligned}
$$

The LO hVP for several other observables, such as the $1 s-2 s$ frequency in positronium and its ground-state HFS interval, is also expressed in terms of $\Pi_{h}^{\prime}(0)$ (see Sect. A for detail). Being too small, the positronium LO hVP contributions are out of our interest here.

Results of the hVP contribution to the Lamb shift in ordinary and muonic hydrogen, published in various recent compilations, are consistent, but based on old and relatively inaccurate calculations such as [4]. Meanwhile, a few of recent and accurate evaluations of the hVP contribution to $a_{e}$ have been available in the literature, which allows us to improve the Lamb shift results (see below). (We remind that our concern is not an accuracy of the calculations by itself, but their reliability (for the individual ones) and their overall consistency (for comparison of the results for different observables).)

To find a value of $\Pi_{h}^{\prime}(0)$, we use calculations of the hVP contribution to $g_{e}-2$ in (11) The latter was recently found in a few publications [2,3]. The results of those direct calculations of the integral over the experimental data for the $a_{e}$ contribution are given in Table 2 . In the same table, we give the extracted value for $\Pi_{h}^{\prime}(0)$ and the related results for the hVP contributions to the Lamb shift in $\mathrm{H}$ and $\mu \mathrm{H}$.

The results from [2] to [3] are in agreement; however, they are based very much on the same data and we cannot treat them as uncorrelated. Because of that we choose their average as a central value and use their individual uncertainty as the uncertainty of the average.

Note, at this stage we discuss a result of 'direct calculations' only, i.e., the result performed with an explicit value of the $R$ function and for a quantity directly related to $\Pi_{h}^{\prime}(0)$.

We have studied above the most simple kernels from Table 1. The other are more complicated. To obtain simple relations, we may rely on their asymptotics. Even $K_{a_{e}}(s)$ has a more complicated shape [see (7)] than it is given in Table 1. However, the dispersion integral starts ${ }^{1}$ with $s_{0}=\left(2 m_{\pi}\right)^{2}$, which leads to a

\footnotetext{
1 That is correct in the leading order in $\alpha$. The channels with photons can be in principle measured in the same way as photonless; however, each additional photon brings a factor of $\alpha$. Therefore, the channels with photons are rather
} 
Table 3 The leading hVP contributions in the limit $m_{\mu}, m_{e} \rightarrow 0$. Here we also include the cases where $K(s)$ is simple and coincides with its asymptotics

\begin{tabular}{lll}
\hline$X_{i}$ & $K_{i: \text { lead }}\left(m_{i}, s\right)$ & $m_{i}$ \\
\hline$a_{\mu}$ & $1 / 3\left(m_{i}^{2} / s\right)$ & $m_{\mu}$ \\
$a_{e}$ & $1 / 3\left(m_{i}^{2} / s\right)$ & $m_{e}$ \\
$\Delta E_{L}(1 s, \mathrm{H})$ & $\left(m_{i}^{2} / s\right)$ & $m_{e}$ \\
$\Delta E_{L}(2 s, \mu \mathrm{H})$ & $\left(m_{i}^{2} / s\right)$ & $m_{\mu}$ \\
$\nu_{\mathrm{Mu}}$ & $\left(m_{i}^{2} / s\right)\left[9 / 2 \ln \left(s / m_{i}^{2}\right)+15 / 4\right]$ & $m_{\mu}$ \\
\hline
\end{tabular}

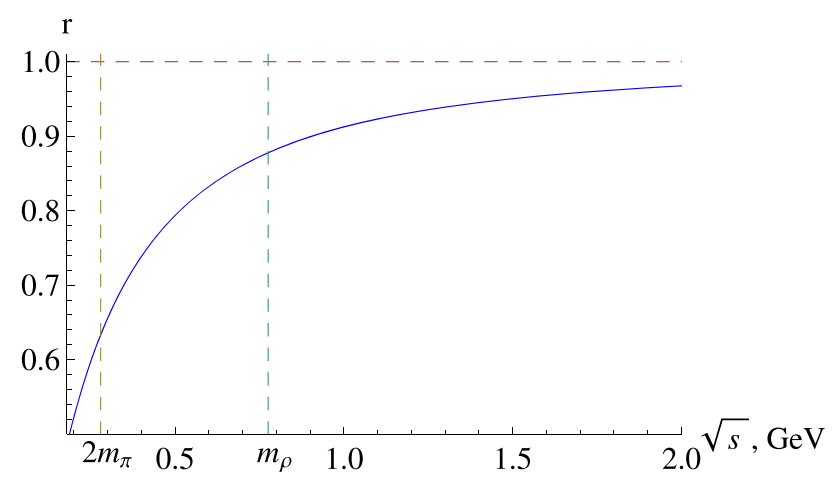

Fig. 4 Kernel ratio $r(s)=K_{a_{\mu}}(s) / K_{a_{\mu} \text { : lead }}(s)$ as a function of $\sqrt{s}$. We remind that $m_{\rho} \simeq 0.78 \mathrm{GeV}, 2 m_{\pi} \simeq$ $0.28 \mathrm{GeV}$

saturation of the asymptotics

$$
K_{\mathrm{a}}(s)=\frac{m_{e}^{2}}{3 s},
$$

at $s \gg m_{e}^{2}$, that is valid for the whole integration area.

The corrections beyond the leading term due to the further $m_{e}^{2} / s$ expansion are below $10^{-5}$ for the all area of the $s$ integration, so we may practically consider the identity (14) as an exact one, since the uncertainty of $R(s)$ is always much higher than $10^{-5}$. For this reason, we have given the expression above as an exact one in Table 1.

A similar equation for the case of $a_{\mu}$ is applicable, but as an approximate one (see Table 3 ). Its accuracy with actual $s$ is roughly at $15 \%$ level for the most of the integration area (see the plot in Fig. 4). The lowest accuracy is at the lowest $s$, close to the $2 \pi$ threshold. The error there is about 35\%. However, that area does not contribute too much to the $s$ integral, which is related to both its central value and the uncertainty.

To understand the accuracy of an approximation for the kernel $K(s)$, we have to look at the function $R(s)$, a convolution with which we are to calculate. Roughly

related to NLO. One of such channels is with $\pi^{0} \gamma$ intermediate states, which starts at $s=m_{\pi}^{2}$. The effect of the $\pi^{0} \gamma$ channel is marginal and is ignored in this paper for the sake of simplicity. In higher orders, there should also be pure photon states produced via hadronic loops which means $s_{0}=0$. Those effects are also negligible for practical applications.

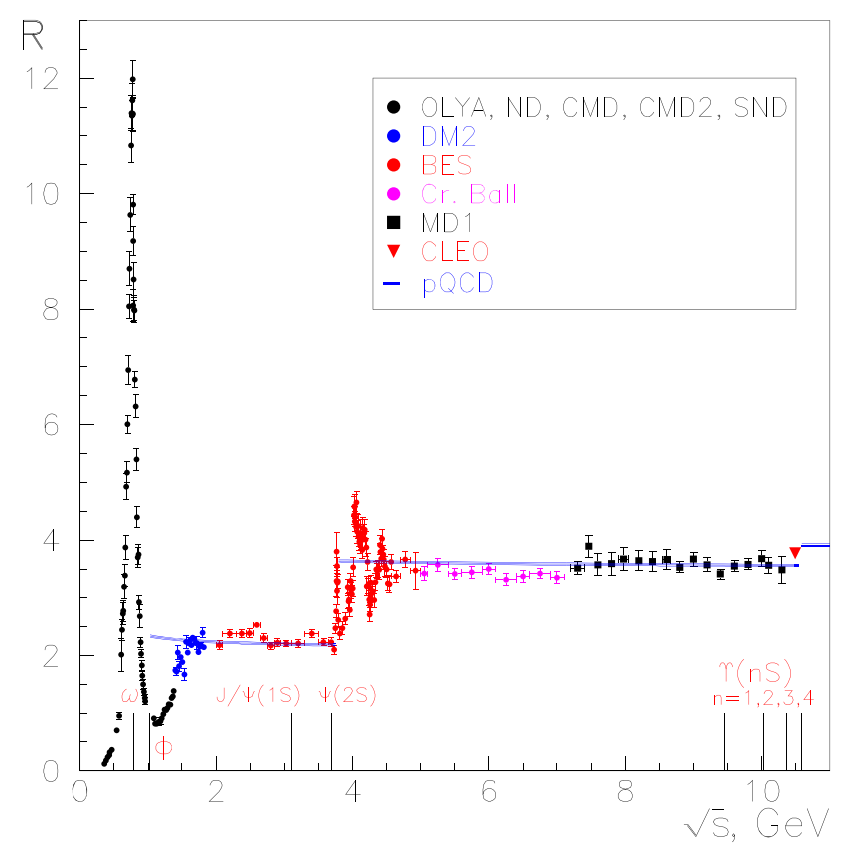

Fig. 5 The $R$-function determined from the electronpositron annihilation to hadrons as published in our paper [5]). The plot was kindly provided to us by Simon Eidelman and we are grateful to him for his permission to use it in [5] and in this paper.)

the behavior of the $R$ function is presented in Fig. 5 . The dominant contributions come from $s \simeq m_{\rho}^{2}$. Note, the $\omega$ and $\phi$ contributions are at $s \approx m_{\rho}^{2}$ from the point of view of the accuracy of the approximation with a smooth-behaving kernel $K$. Meanwhile $\left(m_{\mu} / m_{\rho}\right)^{2} \simeq$ 0.02 which does not make the limit $m_{\mu} \rightarrow 0$ sufficient for accurate calculations, but still makes it useful for a consideration of the correlations between different contributions.

The contribution from the $\rho$-meson $\left(m_{\rho^{0}}=775.26\right.$ (25) MeV; $\Gamma_{\rho}=147.8(9) \mathrm{MeV}$ [13]) as well as from the $\omega\left(m_{\omega}=782.65(12) \mathrm{MeV} ; \Gamma_{\omega}=8.49(8) \mathrm{MeV}\right)$ and $\phi\left(m_{\phi}=1019.461(16) \mathrm{MeV} ; \Gamma_{\phi}=4.249(13) \mathrm{MeV}\right)$ mesons, which come from the same area of $s$, strongly dominates in both the LO hVP contribution to $a_{\mu}$ and its uncertainty (see, e.g., [1]).

Comparing the kernel $K_{a_{\mu}}$ and its asymptotics (see Fig. 4), we find that except of the area essentially below the $\rho$ meson mass, the difference between the kernel 
$K_{a_{\mu}}$ and its asymptotics $K_{a_{\mu} \text { : lead }}$ can be described as

$$
K_{a_{\mu}}(s)=(0.83 \pm 0.13) K_{a_{\mu}: \text { lead }}(s) .
$$

A use of $K_{a_{\mu} \text { : lead }}$ allows us to express any $a_{\mu}$ (LO hVP) result in terms of $\Pi_{h}^{\prime}(0)$. However, the accuracy of (15) may be not entirely sufficient for the whole area of $s$ integration. We have to specifically consider an area of low $s$ where the approximation is not that accurate. Another area where $15 \%$ in (15) is not sufficient is related to very high $s$. The ratio at $s \rightarrow \infty$ is unity; however, the essential result comes from $s$ in a wide area around $m_{\rho}^{2}$ for which the unity is not achievable.

As is well known for a while, the experimental result on $a_{\mu}[7]$ does not agree with theory (see [1] for detail). If any corrections to theory should be done, they are to be small. We intend to apply the relation in (15) rather for corrections to results (or differences between the results) than to the complete values. We are interested in the area below $\sqrt{s}=2 \mathrm{GeV}$. A reason for that is that above this value one can not only measure $\rho(s)$, but also calculate it within pQCD $[14,15]$. The results are in good agreement (see, e.g., $[16,17])$. We do not expect any big corrections there.

Applying that relation to the corrections should only allow us to maintain a high accuracy of the results. Since we consider possible corrections to theory, we have to choose theoretical values which will be "corrected', i.e., our starting point. There are somewhat different theoretical results, but for analyzing the shifts it is not important which one to use. We take advantage that the accurate calculations were performed in [3] on $a_{\mu}(\mathrm{LO} \mathrm{hVP})$ and $a_{e}(\mathrm{LO} \mathrm{hVP})$ in a consistent way and utilize the related results from [3]

$$
\begin{aligned}
& \Delta a_{\mu}(\mathrm{LO} \mathrm{hVP})=689.5(3.3) \times 10^{-10} \\
& \Delta a_{e}(\mathrm{LO} \mathrm{hVP})=1.849(11) \times 10^{-12}
\end{aligned}
$$

as our starting point. We remind that the latter one corresponds to $\Pi_{h}^{\prime}(0) m_{e}^{2}=1.028(6) \times 10^{-6}(\mathrm{cf}$. Table 2$)$.

Denoting a shift (from the reference values) as $\delta$, we find

$$
\begin{aligned}
\delta \mathcal{I}_{a_{e}} & =(1.20 \pm 0.15)\left(\frac{m_{e}}{m_{\mu}}\right)^{2} \delta \mathcal{I}_{a_{\mu}}, \\
\delta \Pi_{h}^{\prime}(0) m_{e}^{2} & =3 \delta \mathcal{I}_{a_{e}} .
\end{aligned}
$$

We intend to apply the relation (15) not to the complete contribution to $a_{\mu}$, which is a convolution with $R(s)$, but to its possible changes due to changes in $R(s)$. Such an approximation on $K(s)$ with focussing on area relatively close to $m_{\rho}$ is valid for relatively large changes in $R(s)$. 'Large change' means either a shift in $a_{\mu}$ larger than or comparable with the uncertainty of $a_{\mu}(\mathrm{hVP})$ or a serious reconsideration of the uncertainty by increasing or decreasing it by a factor, say, of two. Probability of all such changes should follow the value of the uncertainty of partial hVP contribution, i.e., by a product of $K(s)$ and the [absolute] uncertainty $R(s)$ [cf. (6)].

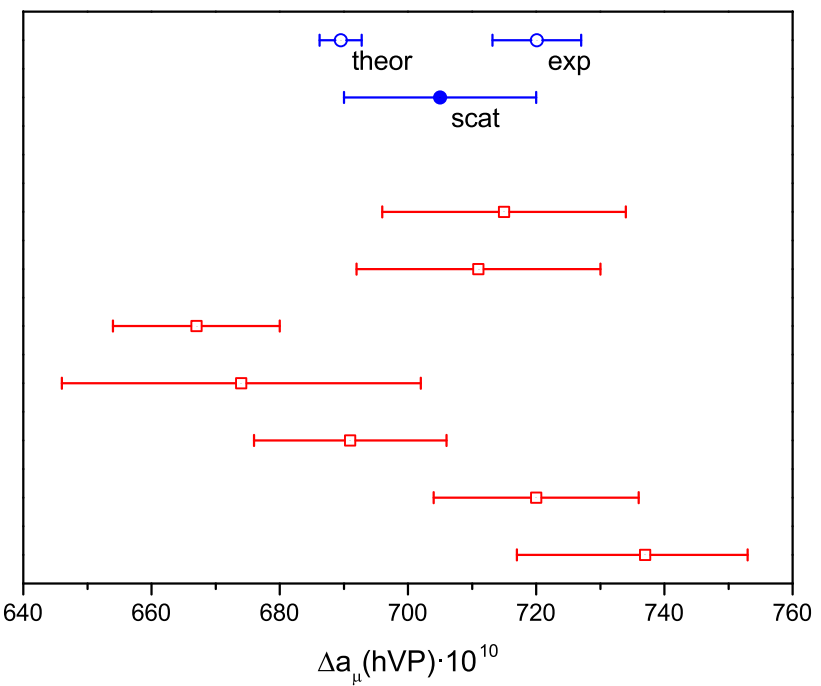

Fig. 6 Direct, indirect, and lattice (with red squares) values for the LO hVP contribution to the anomalous magnetic moment of a muon. The lattice results are summarized in Table 5

The area, which dominates in the uncertainty budget, is expected to be the most probable area from which the change would come. That validates the approximation. Once we consider a small change in $a_{\mu}$, it may come from any area including those where the approximation in (15) is not applicable.

\section{The $a_{\mu}$ discrepancy and its consequences for various LO hVP}

The main motivation to obtain the approximate relations above is the discrepancy in determination of $a_{\mu}$. One can interpret them as a discrepancy between a theoretical result, where the uncertainty comes from hadronic effects, and an experimental one [7], which may be considered as an indirect determination of the hadronic contribution.

Theoretical predictions for the LO hVP contribution to the anomalous magnetic moment of a muon have a certain scatter (see, e.g., $[1,3,18]$ ). The scatter is essentially smaller than the discrepancy of theory and experiment and we ignore it here. As a theoretical value, we use the result in (16) obtained in [3], which is chosen by us because in that publication a result on $\Delta a_{e}(\mathrm{LO}$ $\mathrm{hVP}$ ) is also presented. As mentioned above, a scatter of theoretical results on $\Delta a_{\mu}(\mathrm{LO} \mathrm{hVP})$ is not our concern in this paper, so we can start with any theoretical result on $\Delta a_{\mu}(\mathrm{LO} \mathrm{hVP})$.

In principle, the hadronic NLO includes an uncertainty from the hadronic light-by-light scattering (hLb L) contribution. In this sense, we can consider the result

$$
\Delta a_{\mu}^{\text {from expt }}(\mathrm{LO} \mathrm{hVP})=720.1(6.9) \times 10^{-10},
$$


Table 4 'Reconstruction' of the LO hVP contributions. 'Scatter' stands for an evaluation based on $\Delta a_{\mu}^{\text {from scat }}(\mathrm{hVP})$, while

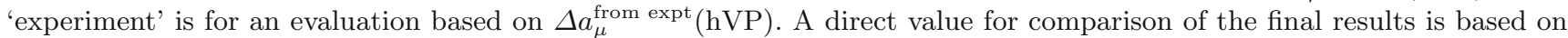
our average in Table 2 . For technical evaluations, we have to start with a direct value for which we know $\Delta a_{\mu}(\mathrm{LO} \mathrm{hVP})$, which is based on results of [3]

\begin{tabular}{llllll}
\hline Comment & $\begin{array}{l}\Delta a_{\mu} \\
{\left[10^{-10}\right]}\end{array}$ & $\begin{array}{l}m_{e}^{2} \Pi_{h}^{\prime}(0) \\
{\left[10^{-6}\right]}\end{array}$ & $\begin{array}{l}\Delta a_{e} \\
{\left[10^{-12}\right]}\end{array}$ & $\begin{array}{l}\Delta E_{L}(1 s) \text { in } \mathrm{H} \\
{[\mathrm{Hz}]}\end{array}$ & $\begin{array}{l}\Delta E(2 p-2 s) \text { in } \mu \mathrm{H} \\
{[\mathrm{meV}]}\end{array}$ \\
\hline Direct (average) & - & $1.033(6)$ & $1.858(11)$ & $-3357(20)$ & $0.01116(7)$ \\
Direct (following [3]) & $689.5(3.3)$ & $1.849(11)$ & $1.028(6)$ & $-3341(20)$ & $0.01111(7)$ \\
Scatter & $705(15)$ & $1.052(25)$ & $1.89(5)$ & $-3419(82)$ & $0.01136(27)$ \\
Experiment & $720(7)$ & $1.076(15)$ & $1.93(3)$ & $-3497(48)$ & $0.01162(16)$ \\
\hline
\end{tabular}

as one extracted from experiment [7] assuming that hLbL is calculated correctly. (To extract $\Delta a_{\mu}^{\text {from }}$ expt (LO hVP) we utilize the QED, NLO hVP, NNLO hVP, and hLbL-scattering contributions as given in [3]).

The most realistic value, however, is neither theoretical nor experimental, but (4)

$$
\Delta a_{\mu}^{\text {from scat }}(\mathrm{LO} \mathrm{hVP})=705(15) \times 10^{-10}
$$

found from scatter of the theoretical and experimental results (see Fig. 6). In the evaluations below, we give values of various hVP contributions, related to direct theoretical calculations; based on $\Delta a_{\mu}^{\text {from expt }}(\mathrm{LO}$ hVP), extracted from experiment, and the one due to the scatter of the data. The latter is the estimation recommended in this paper.

On base of the approximate relations between the LO hVP contributions to $a_{\mu}$ and $a_{e}$ [see (15)] and exact relations between the LO hVP contributions to $a_{e}, \Pi_{h}^{\prime}(0)$, and to the Lamb shift in $\mathrm{H}$ and $\mu \mathrm{H}$ [see (10), (11), (12), (13), and Table 2], we find the results for the LO hVP contribution to various observables as summarized in Table 4 and Fig. 7.

Note that there is no consensus on the theoretical value. Results from scan over $s$ in $e^{+} e^{-}$annihilation are currently less accurate (around the $\rho$-meson mass etc.). The data on $\tau$-decay should be corrected due to violation of the isotopic invariance (introduction of which in [20] explained in the principle then discrepancy between decay data and annihilation by a $\rho^{(0)}-\gamma$ mixing). However, there exist a few different parameterizations for such a correction, which lead to somewhat controversial results [21-23]. Overall analysis sometimes utilizes $\tau$-decay data (see, e.g., $[3,23]$ ), while in other cases it does not (see, e.g., $[18,24]$ ).

Use of recent and more accurate initial-state-radiation (ISR) data shows a certain discrepancy between different experiments (see, e.g., ISR data for the $\pi^{+} \pi^{-}$channel from KLOE [25-27], BaBar [28], and BESIII [29] and their comparison in [18]). Nevertheless, the scatter of theoretical data is essentially smaller than the discrepancy of theory with the experiment. Currently, it is not that important which theoretical value is used as input as far as eventually we plan to calculate the value of $\Delta a_{\mu}^{\text {from scat }}(\mathrm{LO}$ hVP).

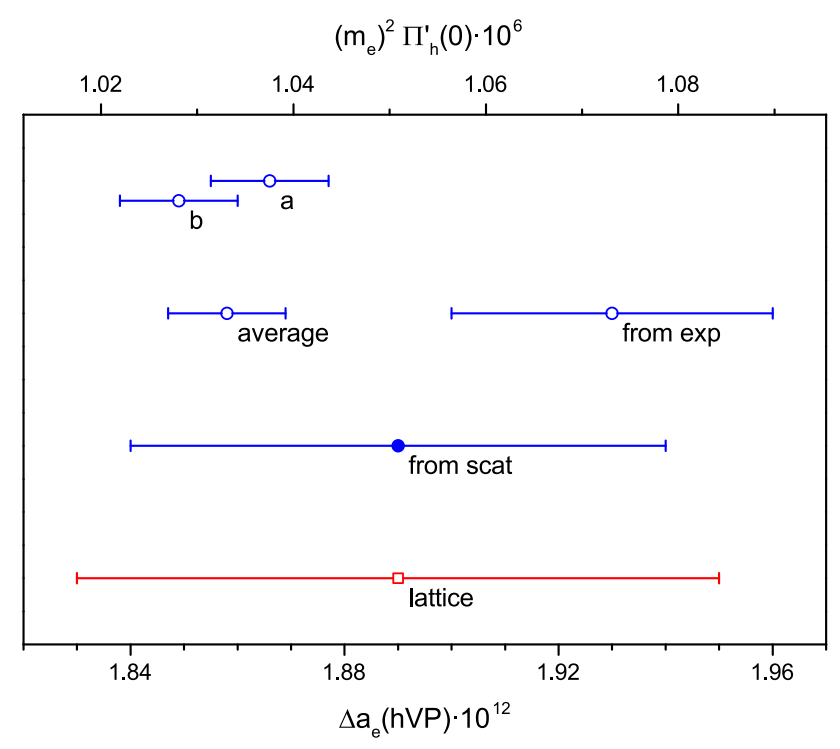

Fig. 7 Direct ( $a$ from [2], $b$ from [3], and average from Table 2), indirect (from experiment and from scatter of theory and experiment), and lattice (from [19]) values for the LO hVP contribution to the anomalous magnetic moment of an electron and to $\Pi_{h}^{\prime}(0)$. The lattice value is discussed below

In our analysis, in this paper we are limited to publications which consider the LO hVP contribution to [at least] two observables. One of such papers [3] presents results on the contributions to $a_{\mu}$ and $a_{e}$ while applying $\tau$-decay data, while the other paper [2], important for our consideration, gives results for $a_{e}$ and Mu HFS interval ignoring $\tau$ data. Consideration of both options made our analysis a representative one.

One has also to mention the result for LO hVP from lattice calculations. Until recently their accuracy was not comparable with that of theory and experiment. Significant progress in the field has produced a situation when the uncertainty of the lattice results is comparable with the uncertainty of $\Delta a_{\mu}^{\text {from scat }}(\mathrm{LO}$ hVP).

The lattice results are summarized in Table 5 . The results are obtained by independent groups but using somewhat similar methods. Below we do not apply the lattice results on $\Delta a_{\mu}(\mathrm{LO} \mathrm{hVP})$ to obtain the $\mathrm{LO} \mathrm{hVP}$ contribution to other variables, but consider them as a 
Table 5 Lattice results on $\Delta a_{\mu}(\mathrm{LO}$ hVP). They are to be compared with $\Delta a_{\mu}^{\text {from scat }}(\mathrm{LO}$ hVP $)=705(15) \times 10^{-10}$ from (4)

\begin{tabular}{ll}
\hline$\Delta a_{\mu}(\mathrm{LO} \mathrm{hVP})$ & Refs. \\
\hline $715(19) \times 10^{-10}$ & {$[30]$} \\
$711(19) \times 10^{-10}$ & {$[19]$} \\
$667(13) \times 10^{-10}$ & {$[31]$} \\
$674(28) \times 10^{-10}$ & {$[32,33]$} \\
$691(15) \times 10^{-10}$ & {$[34]$} \\
$720(16) \times 10^{-10}$ & {$[35]$} \\
$737_{-20}^{+16} \times 10^{-10}$ & {$[36]$} \\
\hline
\end{tabular}

confirmation that $\Delta a_{\mu}^{\text {from scat }}(\mathrm{LO} \mathrm{hVP})$, which we utilize here, is a reasonable estimation. Once the lattice results on $a_{\mu}$ will reach a somewhat higher accuracy, they may be applied to other QED observables along with the technique that was developed in this paper.

There is also a lattice calculation for $\Delta a_{e}(\mathrm{LO} \mathrm{hVP})$. The result [19] of $1.89(6) \times 10^{-12}$ is in perfect agreement with a result from scatter in Table 4 (see also Fig. 7).

\section{LO hVP contribution to muonium HFS interval}

Tables 1 and 3 on the LO hVP contributions contain results for a number of observables considered above in detail. One of important QED observables has not yet been discussed. This remaining case is considered here. It is for the muonium $1 s$ HFS interval. Prior a discussion of the LO hVP term, we have to mention the leading NR contribution to the HFS interval, which is called the Fermi energy. It has several slightly different definitions, such as

$$
\begin{aligned}
E_{F} & =\frac{8}{3}(Z \alpha)^{4} \frac{m_{r}^{3}}{m_{e} m_{\mu}}\left(1+a_{e}\right)\left(1+a_{\mu}\right) \\
\widetilde{E}_{F} & =\frac{8}{3}(Z \alpha)^{4} \frac{m_{r}^{3}}{m_{e} m_{\mu}}
\end{aligned}
$$

where the upper version of the Fermi energy is a result of NR interaction of two full magnetic moments of an electron and a muon, while the one in the bottom is with their Dirac's values. It is convenient to consider the former as the leading term, while expressing the recoil effect (including $\mathrm{hVP}$ which is one of them) in units related to the latter. (That is not important for LO hVP, but a difference in definitions of Fermi energy as a prefactor for $\mathrm{hVP}$ affects definition of NLO hVP.)

Indeed, the anomalous magnetic moments of leptons have their hVP contributions of order $\alpha^{2}$ and the related contributions to the muonium HFS interval would be $\alpha^{2}(Z \alpha)^{4} m_{e}$ with various suppression of the mass factors. However, once the leading NR term, $E_{F}$, is calculated with using the actual values of $a_{e}, a_{\mu}$,

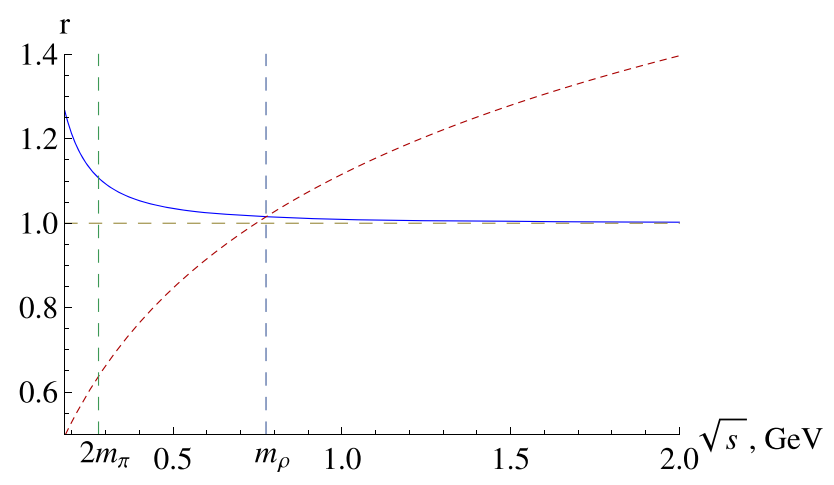

Fig. 8 Kernel ratios $r=K_{\mathrm{Mu}} / K_{\mathrm{Mu} \text { lead }}$ (red) and $r_{0}=$ $K_{\mathrm{Mu}} / K_{\mathrm{Mu}: 0}$ (blue) as a function of $\sqrt{s}$. The related asymptotics are defined in (21) and (22). We remind that $m_{\rho} \simeq$ $0.78 \mathrm{GeV}, 2 m_{\pi} \simeq 0.28 \mathrm{Gev}$

those contributions are included in $E_{F}$ and we are interested in finding the hVP contributions beyond them.

The related LO hVP contribution to the muonium $1 s$ HFS interval is given in terms of (6) and (1) in Table 1 [see also (8)] [11]. It is of order $\alpha(Z \alpha)^{5} m_{e}$ with a characteristic suppression mass factor of $\left(m_{e} / m_{\rho}\right)^{2}$. The related asymptotics at $s \gg m$ is of the form

$$
K_{\mathrm{Mu}: \mathrm{lead}}(s)=\frac{m_{\mu}^{2}}{s}\left[\frac{9}{2} \ln \frac{s}{m_{\mu}^{2}}+\frac{15}{4}\right] .
$$

The results at the limit of $m_{i} \rightarrow 0$ are summarized in Table 3 and one can note that the asymptotics in (21) has a logarithmically enhanced term in contrast to all the other asymptotics considered above [cf. (14) and (9)]. That does not allow us to easily relate the results for the hVP contributions to $a_{\mu}, a_{e}$ and to the Lamb shift with that for the Mu HFS interval. To find such relations, we need an additional simplifying approximation.

To make such, we note that the dominant contribution comes from an area around $s=m_{\rho}^{2}$ (see Fig. 5). Let us introduce

$$
K_{\mathrm{Mu}: 0}(s)=\frac{m_{\mu}^{2}}{s}\left[\frac{9}{2} \ln \frac{m_{\rho}^{2}}{m_{\mu}^{2}}+\frac{15}{4}\right] .
$$

The expression is less accurate as an approximation compared to the one in (21); however, it is easily related to the asymptotics of other kernels [cf. (14) and (9)]. As to the error of the approximation, it is plotted in Fig. 8. Near the $2 \pi$ threshold the error is about 30 $35 \%$ because of higher-order terms of $\mathrm{m}^{2} / \mathrm{s}$ expansion. At $\sqrt{s}=2 \mathrm{GeV}$ the error also reaches that level because of the substitution of

$$
\ln s \rightarrow \ln m_{\rho}^{2}
$$

The contribution from the area above $\sqrt{s}=2 \mathrm{GeV}$ to the Mu HFS interval is not very large (about 20\%) 
Table 6 HVP integrals $\mathcal{I}$ for $a_{\mu}, a_{e}$ and Mu HFS interval. Here, $\mathcal{I}_{a_{\mu}}^{(0)}=\left(m_{\mu} / m_{e}\right)^{2} \mathcal{I}_{a_{e}}$ is the integral related to $K_{\text {a:lead }}\left(m_{\mu}, s\right)$. Values in roman follow the original calculations in $[2,3]$ or experimental result in [7]; the italic ones are from combinations of original data including the evaluation in this paper

\begin{tabular}{lllll}
\hline Ref. & $\mathcal{I}_{a_{\mu}}$ & $\mathcal{I}_{a_{\mu}}^{(0)}$ & $\mathcal{I}_{a_{e}}$ & $\mathcal{I}_{\mathrm{Mu}}$ \\
\hline$[3]$ & $1.278(6) \times 10^{-2}$ & $1.465(9) \times 10^{-2}$ & $3.427(20) \times 10^{-7}$ & - \\
{$[2]$} & - & $1.479(9) \times 10^{-2}$ & $3.458(20) \times 10^{-7}$ & $1.001(6)$ \\
\hline Direct & - & $1.472(9) \times 10^{-2}$ & $3.443(20) \times 10^{-7}$ & $0.997(14)$ \\
$a_{\mu}$, expt & $1.335(13) \times 10^{-2}$ & $1.533(21) \times 10^{-2}$ & $3.586(49) \times 10^{-7}$ & $1.034(18)$ \\
Scatter & $1.306(28) \times 10^{-2}$ & $1.499(36) \times 10^{-2}$ & $3.507(85) \times 10^{-7}$ & $1.013(23)$ \\
\hline
\end{tabular}

and the $R$ function is well described by a step function obtained from perturbative QCD $[14,15]$.

The latter circumstance makes reliable the high- $s$ end of the $s$ integration in the dispersion integral, because of consistency between experimental data and perturbative QCD theory. Due to that, we expect that the major shift in $R(s)$ (if any) may happen between the $2 \pi$-threshold and, say, $\sqrt{s}=2 \mathrm{GeV}$. That allows us to write for the difference between a result of a direct calculation and one with a true $R(s)$ function, based on certain value of $a_{\mu}$ (which may be obtained from experiment, estimated from scatter of experiment and theory, or taken from a lattice calculation), as an indirect estimation, the following

$$
\delta \mathcal{I}_{\mathrm{Mu}}=\left[\frac{27}{2} \ln \frac{m_{\rho}^{2}}{m_{\mu}^{2}}+\frac{45}{4}\right](1.1 \pm 0.4) \delta \mathcal{I}_{a_{\mu}}
$$

We remind that we are interested in relatively large changes in $a_{\mu}$ (see discussion above).

As 'reference' points (to calculate shift $\delta \mathcal{I}_{\mathrm{Mu}}$ ) we consider the results from [2] to [3]. In [2] they found $a_{e}(\mathrm{LO} \mathrm{hVP})$ and $\nu_{\mathrm{Mu}}(\mathrm{LO} \mathrm{hVP})$, which allows one to relate a result on $\mathcal{I}_{a_{e}}$ to the corrected value of $\mathcal{I}_{\mathrm{Mu}}$. Since (23) sets a relation between shifts in $\mathcal{I}_{a_{\mu}}$ and $\mathcal{I}_{\mathrm{Mu}}$, we have to find first a shift in $\mathcal{I}_{a_{\mu}}$ from a known shift in $\mathcal{I}_{a_{e}}$. The results of [3], which include $\Delta a_{e}(\mathrm{LO} \mathrm{hVP})$ and $\Delta a_{\mu}(\mathrm{LO} \mathrm{hVP})$, are suitable for that (see the previous section for details). In terms of the $\mathcal{I}$ integrals, the results of our evaluations are summarized in Table 6.

The results due to the $a_{\mu}$-discrepancy are

$$
\begin{array}{ll}
\Delta \nu_{\mathrm{Mu}}(\mathrm{LO} \mathrm{hVP})=240(4) \mathrm{Hz} & \text { for expt } \\
\Delta \nu_{\mathrm{Mu}}(\mathrm{LO} \mathrm{hVP})=236(5) \mathrm{Hz} & \text { for scatter } .
\end{array}
$$

\section{NLO hVP contribution to the Lamb shift in ordinary and muonic hydrogen}

The NLO hVP contributions to a number of QED observables are known, but not to all of them. In particular, the NLO contribution for $a_{\mu}$ is known $[23,37]$ (see also, e.g., [1]) as well as the ones for $a_{e}$ (see, e.g., $[2,3,37]$ ) and $\mathrm{Mu}$ HFS interval $[38,39]$. (For some variables such as $a_{\mu}, a_{e}$ even NNLO hVP is known [40]; for $a_{\mu}, a_{e}$, and $\mathrm{Mu}$ hfs interval, the hLbL contributions are of the same orders as NLO hVP and needed for a complete NLO hadronic term (see, e.g., [1,3,23,41,42] for detail).)

Here we consider the NLO hVP contribution to the Lamb shift in ordinary and muonic hydrogen. NLO in $\mathrm{H}$ and $\mu \mathrm{H}$ includes a term, which is due to the twoloop vertex with one of those two loops being hVP (see Fig. 9). It is of relative order $\alpha$ compared to the related LO hVP term. In the case of the Lamb shift of an $n s$ state in hydrogen that is a complete NLO correction.

That NLO term is of order $\alpha^{2}(Z \alpha)^{4} m\left(m / m_{\rho}\right)^{2}$. It comes from a slope of the Dirac form factor $F_{1}^{\prime}(0)$ at zero momentum transfer and from the value of the Pauli form factor $F_{2}(0)$ at zero momentum due to the mentioned two-loop vertex, depicted in Fig. 9. The latter, $F_{2}(0)$, is the anomalous magnetic moment of the orbiting lepton. This contribution relates to LO hVP to the related anomalous magnetic moment and it is indeed known. However, since two contributions to the Lamb shift of an $s$ state (due to $F_{1}^{\prime}(0)$ and $F_{2}(0)$ ), which are technically very similar, we consider both of them as a single corrections within the same formalism (see below).

There is also a contribution to the Lamb shift at $l \neq 0$ in ordinary and muonic hydrogen of the same order as the mentioned NLO hVP contribution for the $s$ states. It is completely described by the anomalous magnetic moment in the same matter as the leading QED term for $l \neq 0$, once we deal with a complete value of the anomalous magnetic moment of the orbiting lepton $a_{L}$

$$
\Delta E_{L}^{(a)}(n l)=a_{L} \frac{(Z \alpha)^{4} m_{r}^{2}}{m} \frac{j(j+1)-l(l+1)-3 / 4}{l(l+1)(2 l+1) n^{3}} .
$$

To find the LO hVP contribution to the Lamb shift of $l \neq 0$ states, which is of order $\alpha^{2}(Z \alpha)^{4} m\left(m / m_{\rho}\right)^{2}$, i.e., of the same order as the NLO hVP contribution for $s$ states, it is sufficient to utilize the LO hVP contribution of the anomalous magnetic moment, $\Delta a_{L}(\mathrm{LO} \mathrm{hVP})$, into the identity above. Since this contribution is known (see above), we do not consider it in this paper and focus on the Lamb shift in $n s$ states.

The integral presentation (in terms of $s$ ) for the vertex contribution is of the same shape in $\mathrm{H}$ and $\mu \mathrm{H}$. While that is a complete result for hydrogen, for its 


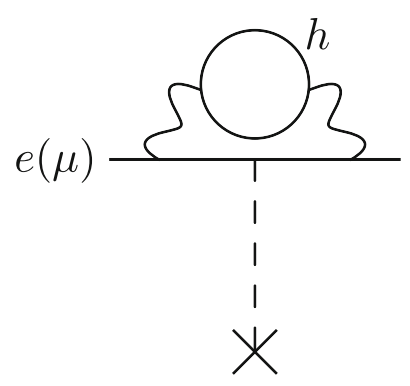

Fig. 9 The NLO hVP contribution to the Lamb shift in $\mathrm{H}$ and $\mu \mathrm{H}$ due to the two-loop vertex
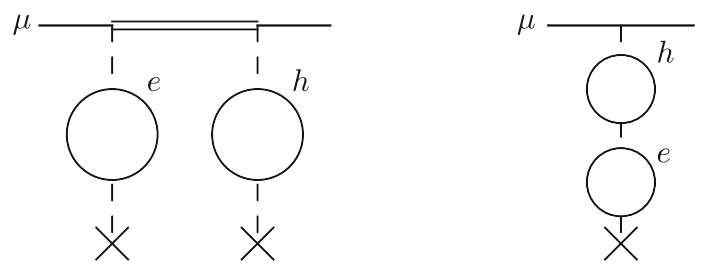

Fig. 10 The Uehling-potential (eVP) correction to the LO hVP contribution. The double horizontal line is for the NR Coulomb Green function of a bound muon. The NLO correction is of order $\alpha^{2}(Z \alpha)^{4} m$

muonic analog we need to take into account other effects as well.

We remind that in $\mathrm{H}$ and $\mu \mathrm{H}$ the $\mathrm{LO}$ hVP contribution to the Lamb shift (see Fig. 3) is proportional to $|\Psi(0)|^{2}$, the squared value of the wave function at origin. In the muonic case, there is a number of different sources for the $\alpha$ correction to $|\Psi(0)|^{2}$ in addition to the two-loop-vertex term discussed above. One of them is from an NR correction to $|\Psi(0)|^{2}$ due to the Uehling potential which is known in a semi-analytic form (see, e.g., [43]), while the other is due to an NR correction with electronic vacuum polarization (eVP) on the same photon as hVP. Those NR specific $\mu$-atom NLO hVP corrections are presented in Fig. 3. The results could be generalized for light two-body muonic atoms. The Uehling correction to the wave function at origin is given for the $1 s$ and $2 s$ states of atoms $A \leq 10$ in [44].

Let us consider now all the mentioned effects in more detail, starting with the two-loop vertex. A two-loop vertex, where one loop is a VP one, delivers pure lepton contributions as well as hVP ones. The simplest leptonic one is the eVP contribution to the Lamb shift in hydrogen, which is well known [45-48]. A similar contribution is due to muonic vacuum polarization $(\mu \mathrm{VP})$ in $\mu \mathrm{H}$ (which can be found with a simple scaling substitution $\left.m_{e} \rightarrow m_{\mu}\right)$. There are also asymmetric in masses pure leptonic contributions, such as eVP in $\mu \mathrm{H}$ and $\mu \mathrm{VP}$ in $\mathrm{H}$, which are known only in part.

Let us find $C_{\text {vert2 }}(m)$ and $K_{\text {vert2 }}(m, s)$ for $s$ states, defined in general in (6), where $m$ is the mass of the orbiting particle. Following [49], we obtain for the Lamb shift of the $n s$ state with an orbiting particle with mass $m$

$$
C_{\text {vert } 2}(m)=24\left(\frac{\alpha}{\pi}\right)^{2} \frac{(Z \alpha)^{4}}{n^{3}} \frac{m_{r}^{3}}{m^{2}}
$$

and

$$
\begin{aligned}
K_{\text {vert } 2}(m, s)= & \frac{1}{288} \int_{0}^{1} \mathrm{~d} x\left[\frac{m^{2} x}{D}\left(6+15 x-13 x^{2}\right)\right. \\
& \left.+\frac{m^{4} x^{3}}{D^{2}}\left(10-7 x+7 x^{2}\right)\right]
\end{aligned}
$$

where

$$
D(x, s)=m^{2} x^{2}+s(1-x) .
$$

The identities above are for the sum of two mentioned earlier contributions (due to $F_{1}^{\prime}(0)$ and $F_{2}(0)$ ).

Here $s$ is a dispersion variable [cf. (5)], which can be introduced both for leptonic and hadronic VP loops. In the leptonic case, we use the parameterization

$$
\frac{1}{q^{2}} \rightarrow \frac{\alpha}{\pi} \int \frac{d v}{1-v^{2}} \frac{v^{2}\left(1-\frac{v^{2}}{3}\right)}{q^{2}+\frac{4 m_{l}^{2}}{\left(1-v^{2}\right)}}
$$

where $m_{l}$ is the lepton mass in the VP loop.

Performing the $x$-integration, we find

$$
\begin{aligned}
K_{\mathrm{vert} 2}(m, s)= & -\frac{1}{72}\left\{\frac{s\left(5 m^{2}-2 s\right)}{m^{2}\left(s-4 m^{2}\right)}+\frac{s^{2}+2 m^{4}}{m^{4}} \ln \frac{s}{m^{2}}\right. \\
& \left.-\frac{s\left(s^{3}-6 s^{2} m^{2}+8 s m^{4}-6 m^{6}\right)}{m^{4}\left(s-4 m^{2}\right)} \cdot J_{10}(s)\right\}
\end{aligned}
$$

where

$$
\begin{aligned}
J_{10}(s)= & \int_{0}^{1} \mathrm{~d} x \frac{m^{2}}{d} \\
= & \frac{1}{\sqrt{s} \sqrt{s-4 m^{2}}} \\
& \times\left\{2 \ln \frac{s+\sqrt{s} \sqrt{s-4 m^{2}}-2 m^{2}}{s+\sqrt{s} \sqrt{s-4 m^{2}}}+\ln \frac{s}{m^{2}}\right\} .
\end{aligned}
$$

It is useful to consider asymptotic behavior at $s \gg$ $m^{2}$. The result reads

$$
K_{\text {vert } 2: \operatorname{lead}}(m, s)=\frac{m^{2}}{s}\left[\frac{1}{36} \ln \frac{s}{m^{2}}+\frac{1}{54}\right] .
$$

Using the kernel obtained above, we can consider various leptonic VP contributions to the Lamb shift of $s$ states in $\mathrm{H}$ and $\mu \mathrm{H}$. Such contributions are eVP and $\mu \mathrm{VP}$ for $\mathrm{H}$ and eVP and $\mu \mathrm{VP}$ for $\mu \mathrm{H}$. The contribution, for which the loop particle and the orbiting one are the same, agrees with the known theoretical result [48]. 


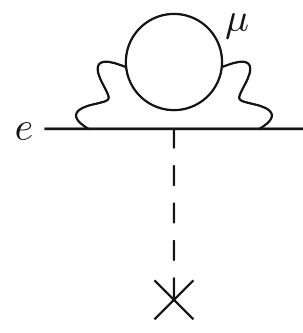

Fig. 11 An 'asymmetric in masses' contribution to the Lamb shift in $\mathrm{H}$ due to the two-loop vertex, where one of the loops is a $\mu \mathrm{VP}$ one

The eVP correction to the Lamb shift in $\mu \mathrm{H}$ reads

$$
\begin{aligned}
\Delta E_{\mathrm{vert}: \mathrm{eVP}}(n s, \mu \mathrm{H})= & \frac{\alpha^{2}(Z \alpha)^{4}}{\pi^{2} n^{3}} \frac{m_{r}^{3}}{m_{\mu}^{2}} \\
\times & {\left[\frac{1}{9} \ln ^{2}\left(\frac{m_{\mu}}{m_{e}}\right)^{2}-\frac{10}{27} \ln \left(\frac{m_{\mu}}{m_{e}}\right)^{2}\right.} \\
& \left.+\frac{2 \pi^{2}}{27}+\frac{85}{162}\right],
\end{aligned}
$$

which agrees with the result from [50].

The $\mu \mathrm{VP}$ correction to the Lamb shift in hydrogen (see Fig. 11), which is a new result, is obtained as

$$
\begin{aligned}
\Delta E_{\mathrm{vert}: \mu \mathrm{VP}}(n s, \mathrm{H})= & \frac{\alpha^{2}(Z \alpha)^{4}}{\pi^{2} n^{3}} \frac{m_{r}^{3}}{m_{e}^{2}}\left(\frac{m_{e}}{m_{\mu}}\right)^{2} \\
& \times\left[\frac{2}{45} \ln \left(\frac{m_{\mu}}{m_{e}}\right)^{2}+\frac{97}{675}\right]
\end{aligned}
$$

which contributes $27 \mathrm{~Hz}$ to the $1 \mathrm{~s}$ Lamb shift in hydrogen and deuterium.

In the case of the NLO hVP, the diagram in Fig. 9 allows us numerical evaluations only. In the case of hydrogen, the expression can be simplified (since $\left.m_{e}^{2} / s \ll 1\right)$

$$
\begin{aligned}
E_{L}^{\mathrm{hVP}: \operatorname{vert} 2}(n s)= & 24\left(\frac{\alpha}{\pi}\right)^{2} \frac{(Z \alpha)^{4} m_{r}^{3}}{n^{3}} \\
& \times \int \frac{\mathrm{d} s \rho(s)}{s}\left[\frac{1}{36} \ln \frac{s}{m_{e}^{2}}+\frac{1}{54}\right]
\end{aligned}
$$

There are different possibilities to obtain a fast preliminary estimation. In particular, we consider two simple methods below. One is to use a simplistic model for the dispersion function $R(s)$, which we have previously used for other occasions (cf. [38,39]). A related result is given in Table 7 . For $\mu \mathrm{H}$, we have applied an exact kernel, since the asymptotics in (32) is not very accurate for $\mu \mathrm{H}$. Therefore, our estimation is obtained with an exact kernel $K(s)$ and approximate function $\rho(s)$.

Another estimation can be obtained with complete $R(s)$ but very approximate $K(s)$. To begin with, we use a rough approximation (cf. (32))

$$
K_{L}^{\mathrm{vert2:approx}}(m, s)=\frac{m^{2}}{s}\left[\frac{1}{36} \ln \frac{m_{\rho}^{2}}{m^{2}}+\frac{1}{54}\right] .
$$

which leads to useful relations

$$
\begin{aligned}
\mathcal{I}_{L}^{\text {vert2:lead }}(\mathrm{H}) & =\left[\frac{1}{12} \ln \frac{m_{\rho}^{2}}{m_{e}^{2}}+\frac{1}{18}\right] \mathcal{I}_{a_{e}}, \\
\mathcal{I}_{L}^{\text {vert2:lead }}(\mu \mathrm{H}) & =\left[\frac{1}{12} \ln \frac{m_{\rho}^{2}}{m_{\mu}^{2}}+\frac{1}{18}\right] \mathcal{I}_{a_{\mu}}^{(0)} .
\end{aligned}
$$

For numerical estimations, which are given in the first line of Table 7, we utilize results from [3] for the righthand side of the identities in (33).

We have obtained two independent estimations for the Lamb shift in both ordinary and muonic hydrogen, and their scatter characterizes the uncertainty of those estimations.

The estimation can be improved once we use an approximation of the kernel as a combination of kernels for Mu HFS interval and for $a_{e}$ (or $a_{\mu}$ ) with the coefficients good for asymptotics $s \gg m^{2}$. In this case, we can use the identity (32) with $\ln \left(s / m^{2}\right)$ without a substitution $s \rightarrow m_{\rho}^{2}$, which is not very accurate for a broad range of $s$.

In particular, one can express the asymptotics of this kernel in terms of asymptotics for the kernels for $a_{\mu}, a_{e}$ and for Mu HFS interval as

$$
\begin{aligned}
K_{L}^{\text {vert2:lead }}\left(m_{e}, s\right)= & \frac{m_{e}^{2}}{m_{\mu}^{2}}\left[\frac{1}{162} K_{\text {Mu:lead }}\left(m_{\mu}, s\right)\right. \\
& \left.+\left(\frac{1}{6} \ln \frac{m_{\mu}}{m_{e}}-\frac{1}{72}\right) K_{\text {a:lead }}\left(m_{\mu}, s\right)\right], \\
K_{L}^{\text {vert2:lead }}\left(m_{\mu}, s\right)= & \frac{1}{162} K_{\text {Mu:lead }}\left(m_{\mu}, s\right) \\
& -\frac{1}{72} K_{\text {a:lead }}\left(m_{\mu}, s\right) .
\end{aligned}
$$

Using the same coefficients, we apply now the relations for the complete kernels, which makes those relations approximate. The result for their integration over $s$ is known (see above) and, following the scatter results, we obtain

$$
\begin{aligned}
\mathcal{I}_{L}^{\text {vert2 }}(\mathrm{H}) & \simeq 4.1 \times 10^{-7}, \\
\mathcal{I}_{L}^{\text {vert2 }}(\mu \mathrm{H}) & \simeq 6.1 \times 10^{-3} .
\end{aligned}
$$

To check the accuracy of (35) and introduce corrections, we have studied the ratios of interest. In previous sections, we were interested in small corrections to known values. It was sufficient to study corrections to $R(s)$ in a relatively narrow area. Here we are to calculate the whole NLO contributions and therefore we are rather interested in the complete area of $s$ integration. 
Table 7 Calculation of the NLO: vert2 hVP contribution to the Lamb shift in $\mathrm{H}$ and $\mu \mathrm{H}$. We give here no uncertainty, but illustrate the scatter of the data obtained by different methods. The uncertainty of NLO hVP can be neglected comparing with the uncertainty of LO hVP. The NLO term rather contributes to the central hVP value, but not to its uncertainty

\begin{tabular}{lcl}
\hline $\mathcal{I}_{L}^{\text {vert2 }}(\mathrm{H})$ & $\mathcal{I}_{L}^{\text {vert2 }}(\mu \mathrm{H})$ & Comment \\
\hline $4.5 \times 10^{-7}$ & $6.0 \times 10^{-3}$ & model from $[38,39]$ \\
$4.4 \times 10^{-7}$ & $5.7 \times 10^{-3}$ & $\ln s \rightarrow \ln m_{\rho}^{2}$ and $\mathcal{I}_{a}$ from $[3]$ \\
$4.0 \times 10^{-7}$ & $5.5 \times 10^{-3}$ & direct \\
$4.2 \times 10^{-7}$ & $5.7 \times 10^{-3}$ & expt \\
$4.1 \times 10^{-7}$ & $5.6 \times 10^{-3}$ & scatter \\
\hline
\end{tabular}

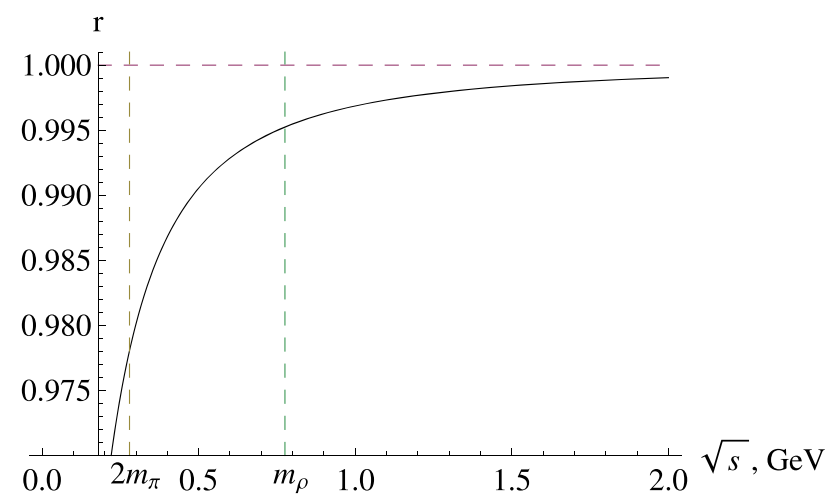

Fig. 12 Kernel ratio $r$ of (36)

The accuracy for the contribution to the Lamb shift in hydrogen is determined by the ratio

$$
\begin{aligned}
& \frac{K_{L}^{\mathrm{vert} 2}\left(m_{e}, s\right)}{\frac{1}{162} \frac{m_{e}^{2}}{m_{\mu}^{2}} K_{\mathrm{Mu}}\left(m_{\mu}, s\right)+\left(\frac{1}{6} \ln \frac{m_{\mu}}{m_{e}}-\frac{1}{72}\right) K_{\mathrm{a}}\left(m_{e}, s\right)} \\
& =0.985 \pm 0.012
\end{aligned}
$$

plotted in Fig. 12 .

For muonic hydrogen, the ratios of interest are (see Fig. 13)

$$
\frac{K_{L}^{\mathrm{vert} 2}\left(m_{\mu}, s\right)}{\frac{1}{162} K_{\mathrm{Mu}}\left(m_{\mu}, s\right)-\frac{1}{72} K_{\mathrm{a}}\left(m_{\mu}, s\right)}=0.92 \pm 0.06
$$

and

$$
\frac{K_{L}^{\mathrm{vert} 2}\left(m_{\mu}, s\right)}{\frac{1}{162} K_{\mathrm{Mu}}\left(m_{\mu}, s\right)-\frac{1}{72} \frac{m_{\mu}^{2}}{m_{e}^{2}} K_{\mathrm{a}}\left(m_{e}, s\right)}=0.93 \pm 0.05
$$

Using the relations above, we obtain central values given in Table 7 . We still do not give any uncertainty. The most uncertain estimation can be obtained from the scatter value. Even its uncertainty is smaller that the uncertainty of the approximate relations we apply. The uncertainty of the NLO: vert 2 term is much smaller than the one of LO hVP and can be ignored.

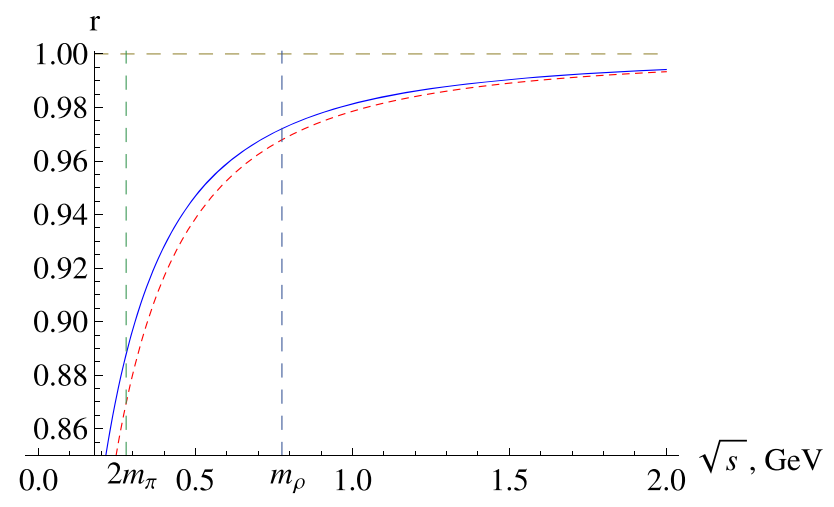

Fig. 13 Kernel ratios $r$ of (37) (red) and (38) (blue)

Finally, for the NLO hVP: vert2 terms we arrive at

$$
\begin{aligned}
\Delta E_{L}^{\mathrm{H}}(1 s) & =18 \mathrm{~Hz} \\
\Delta E_{L}^{\mu \mathrm{H}}(2 p-2 s) & =-0.02 \mu \mathrm{eV} .
\end{aligned}
$$

\section{The hVP contribution to the Lamb shift in hydrogen and muonic hydrogen}

The NLO hVP contributions have been known for $a_{\mu}$ (see, e.g., [18]), $a_{e}$ (see, e.g, [2]), and Mu HFS interval $[38,39]$, but not for the Lamb shift in both ordinary and muonic hydrogen. In principle, any accurate evaluation of LO should be accompanied with an NLO calculation, since the LO uncertainty is often comparable to the NLO contribution. The NLO contributions to Lamb shift in $\mathrm{H}$ and $\mu \mathrm{H}$ have been already in part considered above.

In the case of hydrogen, NLO consists of the vert2 term only. It is of relative order of $\alpha$ to the LO hVP term. A contribution with an extra $Z \alpha$ exists, but it has an additional suppression factor of $m_{e} / m_{\rho}$. Theory of $\mathrm{hVP}$ for the $1 s$ Lamb shift in hydrogen is summarized in Table 8. We consider NLO not as the result by itself, but rather to compare it with the uncertainty of LO. For the $1 s$ state in hydrogen it is $18 \mathrm{~Hz}$.

The NLO contribution is the same (in units of the LO term) for any $s$ state. Since the NLO contribution has an additional factor of $\alpha$, it is the same in units of the 
Table 8 The hVP contribution in hydrogen. We use here the results based on scatter of $a_{\mu}$ data

\begin{tabular}{lll}
\hline Term & Order & Contribution to $E_{L}(1 s)[\mathrm{Hz}]$ \\
\hline Leading & $\alpha(Z \alpha)^{4} m\left(m_{e} / m_{\rho}\right)^{2}$ & $-3419(82)$ \\
NLO & $\alpha^{2}(Z \alpha)^{4} m\left(m_{e} / m_{\rho}\right)^{2}$ & 18 \\
\hline Total & & $-3401(82)$ \\
\hline
\end{tabular}

Table 9 LO and NLO hVP contributions to the $2 p-2 s$ Lamb shift in $\mu \mathrm{H}$, based on scatter estimations. See Sect. B in Appendix for detail

\begin{tabular}{ll}
\hline Term & $\begin{array}{l}\Delta E_{L}(2 p-2 s) \\
{[\mu \mathrm{eV}]}\end{array}$ \\
\hline LO hVP & $11.36(27)$ \\
NLO hVP (vert2) & -0.02 \\
NLO hVP (Ueh- $\psi)$ & 0.04 \\
NLO hVP (Ueh-kern) & 0.05 \\
\hline total hVP & $11.43(27)$ \\
\hline
\end{tabular}

LO term for all the low- $Z$ H-like atoms. In particular, for an arbitrary $s$ state of a low- $Z$ H-like atom it is

$$
18 \mathrm{~Hz} \times \frac{Z^{4}}{n^{3}} .
$$

The LO hVP term also scales as $Z^{4} / n^{3}$.

Theory of the Lamb shift in muonic hydrogen is somewhat different from that in hydrogen. In particular, additionally to the vert2 term there are NR Uehling contributions. One of them is due to a modification of the wave function at origin, that has been considered for a number of occasions (see the left diagram in Fig. 10). The other one is with eVP on the same photon as hVP (see the right diagram in Fig. 10). Both are similar to the corrections in muonic atoms for HFS interaction and for finite-nuclear-size contributions (FNS) to the Lamb shift (cf., e.g., [44,51]). We apply here the related results following [44]. The evaluation of soft contributions due to the Uehling potential is given in Appendix in Sect. B for the nuclear charge $Z=1-10$.

Summary on theory of hVP for the $2 p-2 s$ Lamb shift in $\mu \mathrm{H}$ is given in Table 9. Similarly to the case of hydrogen above, here we consider NLO not as the result by itself, but rather to compare it with the LO uncertainty. The results on other low- $Z$ two-body muonic atoms are given in Appendix in Sect. B.

As mentioned in a number of publications (see, e.g., $[4,5,52])$, there is a certain interference in treatment of the FNS and hVP contributions to the Lamb shift in $\mathrm{H}$ and $\mu \mathrm{H}$ (and other atoms). In a number of situations, such as, e.g., in determination of the form factors, the $\mathrm{hVP}$ correction is not subtracted while evaluating the scattering data and therefore instead of form factors one measures rather

$$
\frac{G\left(q^{2}\right)}{1-\frac{\alpha}{\pi} \frac{\Pi_{h}\left(q^{2}\right)}{q^{2}}} .
$$

For the expansion at low $q^{2}$, that means that instead of the charge radius squared we effectively deal with

$$
-\frac{1}{6} R_{p}^{2}+\frac{\alpha}{\pi} \Pi_{h}^{\prime}(0)
$$

If only the LO hVP contribution to the Lamb shift of either ordinary or muonic atom is important, it is more advantageous not to consider hVP explicitly at all, which means an effective redefinition of the rms nuclear charge radius following (40).

Mathematically speaking, FNS and hVP do not make a single block. While fitting, they have a somewhat different structure and it may be good to separate them. Corrections, e.g., a soft QED correction for $\mu$ atoms, are also not of exactly the same form. The combinatoric coefficient is different (see Sect. B in Appendix). A similar situation is with the radiative corrections to electron-proton scattering, e.g., eVP corrections for one-photon exchange have a different (by a factor of two) coefficient for form factor and for hVP. Because of logarithmic enhancement for $q^{2} \gg m_{e}^{2}$, such a correction can be more important for scattering than for the bound problem.

In the case of light compound nuclei (deuteron, triton, helion, $\alpha$-particle), the situation is more tricky. The radius can be determined either from muonic atoms or from scattering. In the meantime, there are various nuclear models (see, e.g., [53]) which may be efficiently used for calculation of the nuclear-structure contributions. A value of the radius used in theory of compound nuclei is usually not the one obtained from the models, but they still have to produce such a radius from the model for a consistency test. The hVP effects are definitely to be added to nuclear-physics-model calculations of the radius. However, at the present level of accuracy that is not really important. The most accurate applications relate $\mathrm{H}$ and $\mu \mathrm{H}$ and their isotopes, where the mentioned effects are rather marginal.

Here, we have calculated hVP corrections in ordinary and $\mu$ atoms and it is up to those who consider their complete theory to include them or not. However, the decision should be explicitly explained in order to avoid confusion and to allow ones to maintain consistency with other calculations. 


\section{Conclusions}

In area of precision low-energy tests of QED, there is a number of discrepancies, such as the proton-radius puzzle and a controversy with $a_{\mu}$. These discrepancies create problems at two different levels. One that attracts most of the attention is roughly speaking a question, which of controversial results are right and which are wrong. In meantime, there is an additional problem in the shadow of the previous one. Presence of a controversy means that certain data and certain standard treatments are compromised. Meanwhile, they are not applied exclusively to the controversial quantities, but are often also used for a number of others.

For instance, the proton-radius puzzle tells us that probably the use of the data on the form factors of a proton in a wide region, as it is usually done, is incorrect. (If it is, it is not clear whether we have a problem with the data or their interpretation, including the fitting 'as usual'.) That means not only that the related value of the proton charge radius is [probably] wrong, but also that the magnetic radius and various other quantities such as the so-called Zemach and Friar radii, cannot be considered as reliable, once they are deduced from the same evaluation that delivers a probably incorrect value of $R_{p}$. The Zemach radius [54] is required to obtain theoretical predictions for the HFS interval in ordinary and muonic hydrogen, while the Friar radius [55-57] is a key element of a presentation of a higherorder FNS contribution, which plays an important role for a determination of $R_{p}$ from the Lamb shift in $\mu \mathrm{H}$. (The related higher-order FNS contribution is essentially smaller than a discrepancy for the radius from $\mu \mathrm{H}$ spectroscopy and $e-p$ scattering, but essentially larger than the uncertainty of $R_{p}$ due to the $\mu \mathrm{H}$ experiment [58] and of a pure QED part of the related theory (see, e.g., [5]).) See [59,60] for more details.

A number of recent indications from hydrogen spectroscopy [61,62] and electron-proton low-momentumtransfer scattering [63] have pointed out that the muonic value of the proton charge radius [58] is in general correct. (Not all the recent results support the muonic value of $R_{p}$, e.g., the spectroscopic result [64] confirms the 'old' hydrogen value, which is consistent with a result of a [former] 'usual' evaluation from $e-p$ scattering [65].) However, they do not help for understanding what to do with the overall scattering data and their fits for the electric form factor, that is required for a calculation of the Friar term in order to eventually obtain a muonic value of $R_{p}$.

The problem with $a_{\mu}$ is somewhat different. If theory is compromised, that means that a calculation of LO $\mathrm{hVP}$ is compromised at the first place. (The [dominant] pure QED part of theory [66] is much more accurate and reliable than the LO hVP contribution, while the higher-order hadronic effects are smaller than LO hVP and therefore also more reliable (see [1] for detail).)

Standard theoretical evaluations of the LO hVP term are based on the dispersion integral in (1) over experimental data (presented with the $R(s)$ function). The same function $R(s)$ is applied for other observables. The discrepancy for $a_{\mu}$ should be considered as the most accurate test of calculations of the LO hVP contribution through a dispersion integral. The discrepancy indicates that the dispersion calculation is compromised. It is the most important not just to update various hVP results from an occasion to an occasion, but to check whether a more recent value is consistent with the previous one, and to be sure that all the results for different observables, applied within a certain analysis, are consistent.

Disagreement between experiment and theory on $a_{\mu}$ opens a question, how the hVP results for $a_{\mu}$ should be interpreted. There are four options: (i) experiment [7] is not correct; LO hVP theory (see, e.g., [3]) is not; the evaluation of hLbL is not; or certain new physics is present.

Technically, the option of new physics as a solution of the discrepancy between theory and experiment leads to the same consequences for the application as a possible [relatively] large error in the hLbL contribution. The experimental value of $a_{\mu}$ is applicable in such a case as well as the $R$ function and all the direct results on the LO hVP contributions to various observables; however, one has to consider an additional contribution to various QED observables, which has not been accounted for (or has not been correctly accounted for) previously. Such a contribution (e.g., due to a conceptual error in the hLbL term or due to new physics) for an observable of interest may happen to be larger than the uncertainties due to LO hVP (theoretical) and $a_{\mu}$ (experimental). In both cases (hLbL and new physics), the contribution is to be model dependent. The required new physics should be introduced with a certain model or one has to consider a completely new approach to hLbL, drastically different from the previous calculations. Eventually, that leads to a bunch of model-dependent corrections to various observables.

Until such a model appears we have to focus our attention on two other options: either the $a_{\mu}$ measurement [7] has a systematic error, or the data on $R(s)$, applied for a standard calculation of LO hVP, have to be reconsidered. The data may involve the measurements errors, the errors in interpretation (such as radiative corrections), or models to extend the data beyond the experimental reach (to low-luminosity kinematic areas and/or channels).

Without new data or a hint, we are not capable to choose between hVP theory or experiment ${ }^{2}$, and therefore as an estimation of the accuracy of LO hVP we consider the scatter of the data from its direct and indirect determination. The estimation, based on the scatter, pretty well agrees with the lattice results [19,30-36] (see Fig. 6). As a consistent set of hVP contributions, we choose the values given in Table 10. They are based on scatter of $a_{\mu}$ (see Fig. 6) and details of their evaluation have been given in the paper.

\footnotetext{
${ }^{2}$ Hopefully, first results expected from Fermilab [67] will soon help to clarify the situation.
} 
Table 10 The hVP contributions to various QED observables. We present LO hVP for most of the contributions, except for the Lamb shift, for which we present $\mathrm{LO}+\mathrm{NLO}$

\begin{tabular}{ll}
\hline Quantity & Value \\
\hline$\Delta a_{\mu}(\mathrm{LO} h V P)$ & $705(15) \times 10^{-10}$ \\
$\Delta a_{e}(\mathrm{LO} h V P)$ & $1.89(5) \times 10^{-12}$ \\
$\Delta \nu_{\mathrm{Mu}}(1 s)(\mathrm{LO} \mathrm{hVP})$ & $236(5) \mathrm{Hz}$ \\
$\Delta E_{L}^{\mathrm{H}}(1 s)(\mathrm{LO}+\mathrm{NLO} \mathrm{hVP})$ & $-3401(82) \mathrm{Hz}$ \\
$\Delta E_{L}^{\mu \mathrm{H}}(2 p-2 s)(\mathrm{LO}+\mathrm{NLO} \mathrm{hVP})$ & $11.4(3) \mu \mathrm{eV}$ \\
$m_{e}^{2} \Pi_{h}^{\prime}(0)$ & $1.052(25) \times 10^{-6}$ \\
$m_{\mu}^{2} \Pi_{h}^{\prime}(0)$ & $4.50(11) \times 10^{-2}$ \\
\hline
\end{tabular}

The muonium 1s HFS interval deserves a somewhat more accurate consideration. Technically speaking, the largest $\mathrm{hVP}$ contribution to Mu HFS interval is not the one considered here as the LO hVP term, but another one due to $a_{\mu}$. They are of the same order in $\alpha$ but have different suppression mass factors, such as $m_{\mu}^{2} / m_{\rho}^{2}$ (for $a_{\mu}$ ) and $m_{e} m_{\mu} / m_{\rho}^{2}$ (for a 'direct' contribution to $\mathrm{Mu}$ HFS). A small LO hVP contribution to $a_{e}$ is also of the same order in $\alpha$ but has a suppression factor of $m_{e}^{2} / m_{\rho}^{2}$.

Once we define the leading NR term of the muonium HFS interval, the Fermi energy $E_{F}$, as the result of interaction of full moments of electron and muon as given in (19), the $a_{\mu}$-related $\mathrm{hVP}$ contribution is a part of the Fermi energy, while the leading hVP correction to it is $\Delta \nu_{\mathrm{Mu}}(\mathrm{LO} \mathrm{hVP})$. However, we have to consider the leading NR term and the LO hVP correction to the Mu HFS interval in a consistent way. Since QED theory of $a_{\mu}$ is set, any uncertainty and error is understood as uncertainty or error of direct or indirect determination of the contribution of hVP effects. A simultaneous inclusion in the same theoretical expression of an experimental value of $a_{\mu}$ from [7] and $\Delta \nu_{\mathrm{Mu}}(\mathrm{LO} \mathrm{hVP})$ from a dispersion integration (1) (such as the result from [2]) is inconsistent.

Our recommended set of consistent values for the involved quantities, based on the scatter for $a_{\mu}$, is

$$
\begin{aligned}
a_{\mu} & =11659193.7(15.4) \times 10^{-10}, \\
\Delta \nu_{\mathrm{Mu}}(\mathrm{hVP}) & =241(5) \mathrm{Hz} \\
r\left(a_{\mu}, \nu_{\mathrm{Mu} \mathrm{hfs}}(\mathrm{hVP})\right) & =0.87
\end{aligned}
$$

where we also give a correlation coefficient.

In Table 10, we present for the Lamb shift in hydrogen and muonic hydrogen a sum LO+NLO hVP, since NLO contribution is an original result of this paper. One may be interested in LO and NLO separately and not only for ordinary and muonic hydrogen, but also for other atoms. The NLO hVP in ordinary atoms and a hard part of the contribution to muonic atoms is discussed in Sect. 5, while the soft part of the muonicatom correction is present in Appendix in Sect. B. Here we summarize the results for $A=1-4$ for ordinary and muonic atoms that are given in Tables 11 and 12, respectively.
One may also be interested in their intercorrelations. As concerns correlations for the Lamb shift of the $n s$ states in ordinary and muonic atoms, all the LO $\mathrm{hVP}$ contributions are proportional to the same value, namely $\Pi_{h}^{\prime}(0)$ and therefore they are correlated with a correlation coefficient $r=1$. (We remind that the $\mathrm{LO}$ $\mathrm{hVP}$ contribution vanishes for $l \neq 0$.)

We do not consider here few-electron atoms, such as $\mathrm{He}$, or simple molecules, such as $\mathrm{HD}^{+}$; however, we expect that LO hVP for most of low-energy variables is to be described with contact interactions (an electron-electron or electron-nucleus one) and therefore it is to be proportional to $\Pi_{h}^{\prime}(0)$, which makes our finding directly applicable for such atomic and molecular systems. The exception is the hyperfine interaction (cf. Sect. 4) for which correlations make a more complicated form.

Having in mind various projects on accurate measurements of pure leptonic properties, such as the anomalous magnetic moment of electron [68] and muon [67, $69]$, energy intervals in muonium $[70,71]$ and positronium $[72,73]$, progress in related theoretical QED calculations [74-78], and in determination of involved fundamental constants (such as the fine structure constant $\alpha[79])$, a better understanding of related hVP contributions, that is one of the limiting factor of theory of pure leptonic systems, is important.

After the work was completed a number of new publications become available [80-86]. They somewhat improve the situation on certain issues but do not affect the conclusions of the paper. One of the results of this paper is the relations between LO hVP contributions to different observable. The data may play a role of starting point. The most important problem with the data is a discrepancy of theoretical and experimental results on $a_{\mu}$. A minor change of a dispersion result for the theoretical value is marginal comparing with the size of the discrepancy. Once we deal with a half sum of theoretical and experimental values as the central value of our prediction and their half difference as an estimation of the uncertainty, any change in actual theoretical values is negligible.

Still, new data-driven evaluations of the LO hVP contribution to $a_{\mu}$ was presented in [80-82]. Two of them $[80,81]$ utilize $e^{+} e^{-}$annihilation data only, while the other [82] uses also the $\tau$-decay data. The consensus on use or non-use of $\tau$ data has not yet reached.

There is a new result on LO hVP from lattice [83].

There has been also a progress in calculation of hadronic LbL contribution [84-86]. Within our logics the latter contributes to the indirect determination of the LO hVP by striping the experimental value of $a_{\mu}$ out of all the other theoretical contributions. The hLbL one is a dominant contribution to the theoretical uncertainty of such a procedure.

Technically, the results from [80] could be useful for realization of our strategy line, because that seems to be the first paper with appropriate description of $R(s)$ where the LO hVP contribution is calculated with the same $R(s)$ for $a_{\mu}, a_{e}$, and Mu HFS. That should sim- 
Table 11 The LO+NLO hVP contribution to the $1 s$ Lamb shift in light $(Z=1,2)$ [ordinary] hydrogen-like atoms

\begin{tabular}{lll}
\hline Atom & LO hVP $[\mathrm{kHz}]$ & LO+NLO hVP $[\mathrm{kHz}]$ \\
\hline${ }^{1} \mathrm{H}$ and ${ }^{2} \mathrm{H}$ & $-3.42(8)$ & $-3.40(8)$ \\
${ }^{3} \mathrm{He}^{+}$and ${ }^{4} \mathrm{He}^{+}$ & $-54.8(1.3)$ & $-54.5(1.3)$ \\
\hline
\end{tabular}

Table 12 The LO+NLO hVP contribution to the $2 p-2 s$ Lamb shift in light $(Z=1,2)$ muonic atoms

\begin{tabular}{lll}
\hline Nucleus & LO hVP $[\mu \mathrm{eV}]$ & LO+NLO hVP $[\mu \mathrm{eV}]$ \\
\hline${ }^{1} \mathrm{H}$ & $11.36(27)$ & $11.43(27)$ \\
${ }^{2} \mathrm{H}$ & $13.28(32)$ & $13.36(32)$ \\
${ }^{3} \mathrm{He}$ & $224(5)$ & $226(5)$ \\
${ }^{4} \mathrm{He}$ & $230(6)$ & $233(6)$ \\
\hline
\end{tabular}

plify the evaluations, however, the eventual results (see Table 10) would not change much.

Acknowledgements The authors are grateful to A. Czarnecki, S.I. Eidelman, V.G. Ivanov, E.Yu. Korzinin, T. Mibe, M. Nio, D. Nomura, K. Pachucki, K. Shimomura, R. Szafron and Th. Udem for useful and stimulating discussions. The work was supported in part by DFG (under grant No. KA 4645/1-1), ERC (under H2020 Grant No. 742247), and RSF (under Grant \# 17-12-01036).

Funding Open Access funding enabled and organized by Projekt DEAL.

Data Availability Statement This manuscript has no associated data or the data will not be deposited. [Authors' comment: All the data we used are published elsewhere and we give the related citations. We present the most important input data with appropriate citations explicitly in our paper, while our output results are also explicitly given in various tables of our paper.].

Open Access This article is licensed under a Creative Commons Attribution 4.0 International License, which permits use, sharing, adaptation, distribution and reproduction in any medium or format, as long as you give appropriate credit to the original author(s) and the source, provide a link to the Creative Commons licence, and indicate if changes were made. The images or other third party material in this article are included in the article's Creative Commons licence, unless indicated otherwise in a credit line to the material. If material is not included in the article's Creative Commons licence and your intended use is not permitted by statutory regulation or exceeds the permitted use, you will need to obtain permission directly from the copyright holder. To view a copy of this licence, visit http://creativecomm ons.org/licenses/by/4.0/.

\section{Appendix A: LO hVP contribution to the positronium energy levels}

There are two types of the LO hVP contributions to positronium (Ps) energy levels. Both are of order $\alpha^{5} m_{e}$. (There is

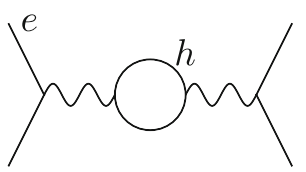

Fig. 14 The LO hVP contribution to the energy of the triplet $S$ state in positronium

also a suppression factor of $\left(m_{e} / m_{\rho}\right)^{2}$, where $m_{\rho}$ represents the scale of hadronic masses in the dispersion integral of (6) where most of the contribution comes from.)

The $\alpha^{5} m_{e}$ hVP contributions are exclusively for the $S$ states, being proportional to the squared value of the wave function at origin. They are also proportional to $\Pi_{h}^{\prime}(0)$, which makes the hVO positronium results to be of interest for this paper.

One of the LO hVP contributions in Ps is a standard Lamb-shift-type term (see Fig. 3) (cf. Table 1; we remind that in positronium $\left.m=m_{e}, m_{r}=m_{e} / 2\right)$

$$
\Delta E(n S)=-\frac{\alpha}{\pi} \frac{\alpha^{4} m_{e}}{2 n^{3}}\left(m_{e}^{2} \Pi_{h}^{\prime}(0)\right) .
$$

The other LO hVP contribution is one to the energy of a triplet $S$ state (see Fig. 14)

$$
\Delta E\left(n^{2 I+1} S_{I}\right)=-\frac{\alpha}{\pi} \frac{E_{F}^{A}}{n^{3}}\left(4 m_{e}^{2} \Pi_{h}^{\prime}(0)\right) \delta_{I 1},
$$

where

$$
E_{F}^{A}=\frac{1}{4} \alpha^{4} m_{e}
$$

is the annihilation-channel contribution to the leading term of the Ps $1 S$ HFS interval. The triplet-state contribution affects both the HFS interval and the energy of center of mass of the $n S$ states.

An accurate result on the LO hVP contribution to the Ps $1 s$ HFS interval was published in [87]. The result is related to

$$
m_{e}^{2} \Pi_{h}^{\prime}(0)=1.027(2)
$$

which has a smaller uncertainty than those based on calculations in [2] and [3] (see Table 2), while the latter have been obtained from more sophisticated evaluations. The central 
Table 13 The numerical results for the wave-function correction for the $1 s$ and $2 s$ states in light muonic atoms $(Z \leq 10)$ (cf. [51,94]) following (B3). See also [44]

\begin{tabular}{lllll}
\hline Nucleus & $\kappa$ & $Z$ & $C_{1}^{(\psi)}(1 s)$ & 1.7312 \\
\hline${ }^{1} \mathrm{H}$ & 1.3562 & 1 & 1.8012 & 1.8256 \\
${ }^{2} \mathrm{H}$ & 1.4284 & 1 & 2.9050 & 1.4043 \\
${ }^{3} \mathrm{H}$ & 1.4542 & 1 & 2.9204 \\
${ }^{3} \mathrm{He}$ & 2.9083 & 2 & 3.6599 \\
${ }^{4} \mathrm{He}$ & 2.9345 & 2 & 3.6648 \\
${ }^{6} \mathrm{Li}$ & 4.4428 & 3 & 4.2067 \\
${ }^{7} \mathrm{Li}$ & 4.4546 & 3 & 4.2097 \\
${ }^{8} \mathrm{Be}$ & 5.9511 & 4 & 4.6392 \\
${ }^{9} \mathrm{Be}$ & 5.9604 & 4 & 4.6412 \\
${ }^{10} \mathrm{~B}$ & 7.4598 & 5 & 4.9963 \\
${ }^{11} \mathrm{~B}$ & 7.4674 & 5 & 4.9977 \\
${ }^{12} \mathrm{C}$ & 8.9684 & 6 & 5.3001 \\
${ }^{13} \mathrm{C}$ & 8.9749 & 6 & 5.3012 \\
${ }^{14} \mathrm{~N}$ & 10.4771 & 7 & 5.5644 \\
${ }^{15} \mathrm{~N}$ & 10.4827 & 8 & 5.5652 \\
${ }^{16} \mathrm{O}$ & 11.9859 & 8 & 5.5659 \\
${ }^{17} \mathrm{O}$ & 11.9909 & 8 & 5.7987 \\
${ }^{18} \mathrm{O}$ & 11.9953 & 9 & 6.0076 \\
${ }^{19} \mathrm{~F}$ & 13.4991 & 10 & 6.0081 \\
${ }^{20} \mathrm{Ne}$ & 15.0035 & 10 & 6.0086 \\
${ }^{21} \mathrm{Ne}$ & 15.0075 & 10 & 3.1920 \\
${ }^{22} \mathrm{Ne}$ & 15.0112 & & 3.69942 \\
\hline
\end{tabular}

Table 14 The numerical results for the Uehling part of the NLO hVP contribution to the $1 s$ and $2 p-2 s$ Lamb shift in light muonic atoms $(Z \leq 10)$ (cf. [44]). We remind that following (B1) the units related to the coefficients are $\alpha / \pi E_{\mathrm{LO}} \mathrm{hVP}(n s)$, while $E_{\mathrm{LO} \mathrm{hVP}}(2 p-2 s)=-E_{\mathrm{LO} h \mathrm{hP}}(2 s)$. Therefore, the NLO correction is of the same sign as the LO hVP term in both the $1 s$ and $2 p-2 s$ cases

\begin{tabular}{lll}
\hline Nucleus & $C_{1}^{\text {hVP: Ueh }}(1 s)$ & $C_{1}^{\text {hVP: Ueh }}(2 p)-C_{1}^{\text {hVP: Ueh }}(2 s)$ \\
\hline${ }^{1} \mathrm{H}$ & 3.4972 & -3.2597 \\
${ }^{2} \mathrm{H}$ & 3.6220 & -3.3663 \\
${ }^{3} \mathrm{H}$ & 3.6658 & -3.4034 \\
${ }^{3} \mathrm{He}$ & 5.5450 & -4.9614 \\
${ }^{4} \mathrm{He}$ & 5.5714 & -4.983 \\
${ }^{6} \mathrm{Li}$ & 6.8297 & -6.0236 \\
${ }^{7} \mathrm{Li}$ & 6.8380 & -6.0306 \\
${ }^{8} \mathrm{Be}$ & 7.7525 & -6.8076 \\
${ }^{9} \mathrm{Be}$ & 7.7575 & -6.8117 \\
${ }^{10} \mathrm{~B}$ & 8.4792 & -7.4424 \\
${ }^{11} \mathrm{~B}$ & 8.4824 & -7.4452 \\
${ }^{12} \mathrm{C}$ & 9.0777 & -7.9773 \\
${ }^{13} \mathrm{C}$ & 9.0801 & -7.9795 \\
${ }^{14} \mathrm{~N}$ & 9.5865 & -8.4404 \\
${ }^{15} \mathrm{~N}$ & 9.5882 & -8.4421 \\
${ }^{16} \mathrm{O}$ & 10.0284 & -8.8487 \\
${ }^{17} \mathrm{O}$ & 10.0298 & -8.8500 \\
${ }^{18} \mathrm{O}$ & 10.0309 & -8.8512 \\
${ }^{19} \mathrm{~F}$ & 10.4201 & -9.2147 \\
${ }^{20} \mathrm{Ne}$ & 10.7690 & -9.5437 \\
${ }^{21} \mathrm{Ne}$ & 10.7699 & -9.5444 \\
${ }^{22} \mathrm{Ne}$ & 10.7706 & -9.5452 \\
\hline
\end{tabular}


value of the result in [87] is reasonable, while the estimation of the theoretical uncertainty in [87] is considered by us rather as an overoptimistic one. Besides, as we explain in this paper, the very use of a purely theoretical result based on the dispersion integration in (1) is questionable because of a discrepancy between theory and experiment on $a_{\mu}$.

We do not discuss numerical values for positronium here. They can be easily obtained from our formulas (see Table 4 values of $\Pi_{h}^{\prime}(0)$ required for (A1) and (A2)). The contributions are negligible comparing with the current experimental accuracy $[72,88-92]$.

\section{Appendix B: NLO hVP contribution to the Lamb shift in two-body muonic atoms with $Z \leq 10$}

Let us make a brief 'parallel' calculation of the Uehling correction to the leading FNS term (following [44]) and the LO $\mathrm{hVP}$ term in a muonic atom. It is useful to present both contributions in the form

$$
\begin{aligned}
& \Delta E_{\mathrm{FNS}: \mathrm{Ueh}}(n l)=\frac{\alpha}{\pi} C_{1}^{\mathrm{FNS}}(n l) \Delta E_{\mathrm{FNS}: \text { lead }}(n s), \\
& \Delta E_{\mathrm{hVP}: \mathrm{Ueh}}(n l)=\frac{\alpha}{\pi} C_{1}^{\mathrm{hVP}}(n l) \Delta E_{\mathrm{hVP}: \text { lead }}(n s),
\end{aligned}
$$

where the total coefficient for an $X$ quantity $(=$ FNS or $\mathrm{hVP}$ ) due to the Uehling potential is

$$
C_{1}^{\mathrm{X} \text { Ueh }}(n l)=C_{1}^{(\psi)}(n l)+C_{1}^{\mathrm{X}: \operatorname{kern}}(n l) .
$$

Here $C_{1}^{(\psi)}(n l)$ is due to the correction to the wave function at origin

$$
\left|\psi_{n s}(0)\right|^{2} \rightarrow\left(1+\frac{\alpha}{\pi} C_{1}^{(\psi)}(n s)\right)\left|\psi_{n s}(0)\right|^{2} .
$$

It is generic for any $\delta$-function kernels, which is the case for the leading terms for FNS and hVP (see [44]). The contribution is present only for the $n s$ states.

The other correction, $C_{1}^{\mathrm{X}: \text { kern }}$ is a correction to the $\delta$ function-like kernel and, in principle, it depends on the kernel. In general, it may not vanish for $l \neq 0$ states in muonic atoms and, in particular, for both FNS and hVP it does not.

The coefficient $C_{1}^{(\psi)}(n l)$ is well known in a semi-analytic form $[43,51,93,94]$ and

$$
\begin{aligned}
& J(z)=-\frac{2 z^{2}}{3} \int_{0}^{1} \frac{y \sqrt{1-y^{2}}\left(y^{2}+2\right)}{(1+y z)^{2}} \ln \frac{y z}{1+y z}, \\
& L(z)=-\frac{4 z^{2}}{3} \int_{0}^{1} \frac{y \sqrt{1-y^{2}}\left(y^{2}+2\right)\left(y^{2} z^{2}+2\right)}{(1+y z)^{4}} \ln \frac{y z}{1+y z} .
\end{aligned}
$$

The numerical results on the coefficient are summarized in Table 13 for the $1 s$ and $2 s$ states. As we already mentioned, $C_{1}^{(\psi)}(n l)=0$ for $l \neq 0$ and, in particular, $C_{1}^{(\psi)}(2 p)=0$.

The correction due to the modification of the contact term (i.e., the kernel of the interaction) is specific for any contact term. In the case of the FNS contribution in order $\alpha(Z \alpha)^{4} m$ [43] (cf. [51,57,95] and [96-102]) the result is of the form

$$
\begin{aligned}
C_{1}^{\text {FNS: kern }}(1 s) & =-\frac{\pi}{3 \kappa^{3}}+\frac{6+\kappa^{2}}{9 \kappa^{2}}+\frac{2-\kappa^{2}+2 \kappa^{4}}{3 \kappa^{3}} \mathcal{A}(\kappa), \\
C_{1}^{\text {FNS: kern }}(2 s) & =-\frac{\pi}{3 \kappa_{2}^{3}}+\frac{24-44 \kappa_{2}^{2}-29 \kappa_{2}^{4}+22 \kappa_{2}^{6}}{36 \kappa_{2}^{2}\left(1-\kappa_{2}^{2}\right)^{2}} \\
& +\frac{8-20 \kappa_{2}^{2}+33 \kappa_{2}^{4}-20 \kappa_{2}^{6}+8 \kappa_{2}^{8}}{12 \kappa_{2}^{3}\left(1-\kappa_{2}^{2}\right)^{2}} \mathcal{A}\left(\kappa_{2}\right), \\
C_{1}^{\text {FNS: kern }}(2 p) & =-\frac{\pi}{3 \kappa_{2}^{3}}+\frac{24-44 \kappa_{2}^{2}+13 \kappa_{2}^{4}-2 \kappa_{2}^{6}}{36 \kappa_{2}^{2}\left(1-\kappa_{2}^{2}\right)^{2}} \\
& +\frac{8-20 \kappa_{2}^{2}+15 \kappa_{2}^{4}}{12 \kappa_{2}^{3}\left(1-\kappa_{2}^{2}\right)^{2}} \mathcal{A}\left(\kappa_{2}\right),
\end{aligned}
$$

where $\mathcal{A}(\kappa)$ has been defined above.

The result for the hVP: kern term is similar to the known FNS: kern one, but has a different combinatoric coefficient

$$
C_{1}^{\mathrm{hVP}: \operatorname{kern}}(n l)=2 C_{1}^{\mathrm{FNS}: \operatorname{kern}}(n l) .
$$

The numerical results for

$$
C_{1}^{\mathrm{hVP}: \mathrm{Ueh}}=C_{1}^{\mathrm{hVP}: \operatorname{kern}}+C_{1}^{(\psi)}
$$

for the $1 s$ Lamb shift and for the $n=2$ one (i.e., for the $2 p-2 s$ interval) in light muonic atoms are given in Table 14.

A complete result for the $\mathrm{hVP}$ contribution ( $\mathrm{LO}+\mathrm{NLO})$, which includes the Uehling correction discussed in this appendix and the 'hard' NLO: vert2 term considered in Sect. 5 (cf. Table 9) for the $2 p-2 s$ Lamb shift in lightest muonic atoms, is summarized in Table 12 of Sect. 7.

$$
\begin{aligned}
C_{1}^{(\psi)}(1 s)= & \frac{\pi\left(\kappa^{2}-2\right)}{2 \kappa^{3}}+\frac{6-8 \kappa^{2}+5 \kappa^{4}}{3 \kappa^{2}\left(1-\kappa^{2}\right)}+\frac{2-4 \kappa^{2}+3 \kappa^{4}-2 \kappa^{6}}{\kappa^{3}\left(1-\kappa^{2}\right)} \mathcal{A}(\kappa)+J(\kappa), \\
C_{1}^{(\psi)}(2 s)= & \frac{\pi\left(3 \kappa_{2}^{2}-26\right)}{3 \kappa_{2}^{3}}+\frac{312-920 \kappa_{2}^{2}+894 \kappa_{2}^{4}-195 \kappa_{2}^{6}+44 \kappa_{2}^{8}}{18 \kappa_{2}^{2}\left(1-\kappa_{2}^{2}\right)^{3}} \\
& +\frac{104-376 \kappa_{2}^{2}+506 \kappa_{2}^{4}-309 \kappa_{2}^{6}+42 \kappa_{2}^{8}-12 \kappa_{2}^{10}}{6 \kappa_{2}^{3}\left(1-\kappa_{2}^{2}\right)^{3}} \mathcal{A}\left(\kappa_{2}\right)+L\left(\kappa_{2}\right),
\end{aligned}
$$

where

$$
\begin{aligned}
\kappa_{n} & =\frac{\kappa}{n}=\frac{Z \alpha m_{r}}{m_{e} n}, \\
\mathcal{A}(z) & = \begin{cases}\frac{\arccos z}{\sqrt{1-z^{2}}}, & z<1, \\
\frac{\ln \left(z+\sqrt{z^{2}-1}\right)}{\sqrt{z^{2}-1}}, & z>1 .\end{cases}
\end{aligned}
$$

\section{References}

1. F. Jegerlehner, The Anomalous Magnetic Moment of the Muon. Springer Tracts in Modern Physics, vol. 274, 2nd edn. (Springer, Berlin and Heidelberg, 2017)

2. D. Nomura, Th. Teubner, Nucl. Phys. B 867, 236 (2013) 
3. F. Jegerlehner, (n.d.) arXiv:1711.06089

4. J.L. Friar, J. Martorell, D.W.L. Sprung, Phys. Rev. A 59, 4061 (1999)

5. S.G. Karshenboim, E.Yu. Korzinin, V.A. Shelyuto, V.G. Ivanov, J. Phys. Chem. Ref. Data 44, 031202 (2015)

6. P.J. Mohr, D.B. Newell, B.N. Taylor, Rev. Mod. Phys. 88, 035009 (2016)

7. G.W. Bennett et al., Phys. Rev. D 73, 072003 (2006)

8. C. Bouchiat, L. Michel, J. Phys. Rad. 22, 121 (1961)

9. V.B. Berestetskii, O.N. Krokhin, A.K. Khlebnikov, Sov. Phys. JETP 5, 761 (1956)

10. S.J. Brodsky, E. de Rafael, Phys. Rev. 168, 1620 (1968)

11. S.G. Karshenboim, V.A. Shelyuto, Phys. Lett. B 517, $32(2001)$

12. A. Czarnecki, S.I. Eidelman, S.G. Karshenboim, Phys. Rev. D 65, 053004 (2002)

13. M. Tanabashi et al. (Particle Data Group), Phys. Rev. D 98, 030001 (2018) and 2019 update

14. L.R. Surguladze and M.A. Samuel, Phys. Rev. Lett. 66 560 (1991), 66, 2416 (1991) (erratum)

15. R.V. Harlander, M. Steinhauser, Comput. Phys. Commun. 153, 244 (2003)

16. V.V. Anashin et al., Phys. Lett. B 753, 533 (2016)

17. V.V. Anashin et al. (KEDR Collaboration) Phys. Lett. B 788, 42 (2019)

18. A. Keshavarzi, D. Nomura, T. Teubner, Phys. Rev. D 97, 114025 (2018)

19. S. Borsanyi, Z. Fodor, C. Hoelbling, T. Kawanai, S. Krieg, L. Lellouch, R. Malak, K. Miura, K.K. Szabo, C. Torrero, and B.C. Toth (Budapest-MarseilleWuppertal Collaboration), Phys. Rev. Lett. 121, 022002 (2018)

20. F. Jegerlehner, R. Szafron, Eur. Phys. J. C 71, 1 (2011)

21. M. Davier, A. Höcker, B. Malaescu, C.Z. Yuan, Z. Zhang, Eur. Phys. J. C 74, 1 (2014)

22. M. Benayoun, P. David, L. DelBuono, F. Jegerlehner, Eur. Phys. J. C 75, 613 (2015)

23. F. Jegerlehner, EPJ Web Conf. 166, 00022 (2018)

24. M. Davier, A. Hoecker, B. Malaescu, Z. Zhang, Eur. Phys. J. C 77, 827 (2017)

25. F. Ambrosino et al. (KLOE Collaboration) Phys. Lett. B 670, 285 (2009)

26. F. Ambrosino et al. (KLOE Collaboration) Phys. Lett. B 700, 102 (2011)

27. D. Babusci et al. (KLOE Collaboration) Phys. Lett. B 720, 336 (2013)

28. B. Aubert et al. (BABAR Collaboration) Phys. Rev. Lett. 103, 231801 (2009)

29. M. Ablikim et al. (BESIII Collaboration) Phys. Lett. B 753, 629 (2016)

30. T. Blum, P.A. Boyle, V. Gülpers, T. Izubuchi, L. Jin, C. Jung, A. Jüttner, C. Lehner, A. Portelli, and J.T. Tsang (RBC and UKQCD Collaborations), Phys. Rev. Lett. 121, 022003 (2018)

31. B. Chakraborty, C.T.H. Davies, P.G. de Oliveira, J. Koponen, G.P. Lepage, R.S. Van de Water, Phys. Rev. D 96, 034516 (2017)

32. F. Burger, X. Feng, G. Hotzel, K. Jansen, M. Petschlies, and D.B. Renner (ETM Collaboration), J. High Energy Phys. 02, 099 (2014)

33. F. Burger, K. Jansen, M. Petschlies, G. Pientka, Eur. Phys. J. C 76, 464 (2016)
34. C.T.H. Davies, C. DeTar, A.X. El-Khadra, E. Gamiz, St. Gottlieb, D. Hatton, A.S. Kronfeld, J. Laiho, G.P. Lepage, Y. Liu, P.B. Mackenzie, C. McNeile, E.T. Neil, T. Primer, J.N. Simone, D. Toussaint, R.S. Van de Water, and A. Vaquero, Arxiv eprint arXiv:1902.04223

35. A. Gérardin, M. Cè, G. von Hippel, B. Hörz, H.B. Meyer, D. Mohler, K. Ottnad, J. Wilhelm, H. Wittig, Phys. Rev. D 100, 014510 (2019)

36. E. Shintani and Y. Kuramashi (PACS Collaboration), Phys. Rev. D 100, 034517 (2019)

37. B. Krause, Phys. Lett. B 390, 392 (1997)

38. V.A. Shelyuto, S.G. Karshenboim, S.I. Eidelman, Phys. Rev. D 97, 053001 (2018)

39. S.I. Eidelman, S.G. Karshenboim, V.A. Shelyuto, Can. J. Phys. 80, 1297 (2002)

40. A. Kurz, T. Liu, P. Marquard, M. Steinhauser, Phys. Lett. B 734, 144 (2014)

41. K. Melnikov, A. Vainshtein, Theory of the Muon Anomalous Magnetic Moment. Springer Tracts in Modern Physics, vol. 216 (Springer, Berlin and Heidelberg, 2006)

42. S.G. Karshenboim, V.A. Shelyuto, A.I. Vainshtein, Phys. Rev. D 78, 065036 (2008)

43. S.G. Karshenboim, U. Jentschura, V.G. Ivanov, G. Soff, Eur. Phys. J. D 2, 209 (1998)

44. S.G. Karshenboim, E.Yu. Korzinin, V.A. Shelyuto, V.G. Ivanov, Phys. Rev. A 98, 062512 (2018)

45. T. Appelquist, S.J. Brodsky, Phys. Rev. Lett. 24, 562 (1970)

46. T. Appelquist, S.J. Brodsky, Phys. Rev. A 2, 2293 (1970)

47. R. Barbieri, J.A. Mignaco, E. Remiddi, Lett. Nuovo Cimento 3, 588 (1970)

48. R. Barbieri, J.A. Mignaco, E. Remiddi, Nuovo Cimento A 6, 21 (1971)

49. M.I. Eides, H. Grotch, Phys. Lett. B 308, 389 (1993)

50. R. Barbieri, M. Caffo, E. Remiddi, Lett. Nuovo Cimento 7, 60 (1973)

51. S.G. Karshenboim, E.Yu. Korzinin, V.G. Ivanov, JETP Lett. 88, 641 (2008)

52. M.I. Eides, H. Grotch, V.A. Shelyuto, Theory of Light Hydrogenic Bound States (Springer, Berlin-HeidelbergNew York, 2007)

53. C. Ji, S. Bacca, N. Barnea, O.J. Hernandez, N. Nevo Dinur, J. Phys. G: Nucl. Part. Phys. 45, 093002 (2018)

54. A.C. Zemach, Phys. Rev. 104, 1771 (1956)

55. J.L. Friar, Ann. Phys. (NY) 122, 151 (1979)

56. L.A. Borisoglebsky, E.E. Trofimenko, Phys. Lett. 81B, 175 (1979)

57. K. Pachucki, Phys. Rev. A 53, 2092 (1996)

58. A. Antognini, F. Nez, K. Schuhmann, F.D. Amaro, F. Biraben, J.M.R. Cardoso, D.S. Covita, A. Dax, S. Dhawan, M. Diepold, L.M.P. Fernandes, A. Giesen, A.L. Gouvea, T. Graf, T.W. Hänsch, P. Indelicato, L. Julien, C.-Y. Kao, P. Knowles, F. Kottmann, E.O. Le Bigot, Y.-W. Liu, J.A.M. Lopes, L. Ludhova, C.M.B. Monteiro, F. Mulhauser, T. Nebel, P. Rabinowitz, J.M.F. dos Santos, L.A. Schaller, C. Schwob, D. Taqqu, J.F.C.A. Veloso, J. Vogelsang, R. Pohl, Science 339, 417 (2013)

59. S.G. Karshenboim, Phys. Rev. D 90, 053012 (2014)

60. S.G. Karshenboim, E.Yu. Korzinin, V.G. Ivanov, V.A. Shelyuto, Phys. Rev. D 91, 073003 (2015)

61. A. Beyer et al., Science 358, 79 (2017) 
62. N. Bezginov, T. Valdez, M. Horbatsch, A. Marsman, A.C. Vutha, E.A. Hessels, Science 365, 1007 (2019)

63. W. Xiong, A. Gasparian et al., Nature 575, 147 (2019)

64. H. Fleurbaey, S. Galtier, S. Thomas, M. Bonnaud, L. Julien, F. Biraben, F. Nez, M. Abgrall, J. Guéna, Phys. Rev. Lett. 120, 183001 (2018)

65. J. Arrington, I. Sick, J. Phys. Chem. Ref. Data 44, $031204(2015)$

66. T. Aoyama, M. Hayakawa, T. Kinoshita, M. Nio, Phys. Rev. Lett. 109, 111808 (2012)

67. J. Mott (on behalf of the Muon $g-2$ experiment), Hyper. Int. 239, 55 (2018)

68. G. Gabrielse, S.E. Fayer, T.G. Myers, X. Fan, Atoms 7, 45 (2019)

69. M. Abe, S. Bae, G. Beer et al., Prog. Theor. Exp. Phys. 2019, $053 \mathrm{C} 02$ (2019)

70. P. Strasser, M. Abe1, M. Aoki et al. (on behalf of the MuSEUM Collaboration), EPJ Web of Conferences 198, 00003 (2019)

71. I. Belosevic, A. Antognini, Yu Bao, A. Eggenberger, M. Hildebrandt, R. Iwai, D.M. Kaplan, K.S. Khaw, K. Kirch, A. Knecht, A. Papa, C. Petitjean, Th.J. Phillips, F.M. Piegsa, N. Ritjoho, A. Stoykov, D. Taqqu, G. Wichmann, Hyp. Inter. 240, 41 (2019)

72. A. Ishida, T. Namba, S. Asai, T. Kobayashi, H. Saito, M. Yoshida, K. Tanaka, A. Yamamoto, Phys. Lett. B 734, 338 (2014)

73. M.W. Heiss, G. Wichmann, A. Rubbia, P. Crivelli, J. Phys.: Conf. Ser. 1138, 012007 (2018)

74. G.S. Adkins, M. Kim, C. Parsons, R.N. Fell, Phys. Rev. Lett. 115, 233401 (2015)

75. M.I. Eides, V.A. Shelyuto, Phys. Rev. D 92, 013010 (2015)

76. T. Aoyama, T. Kinoshita, M. Nio, Phys. Rev. D 97, $036001(2018)$

77. S. Volkov, Phys. Rev. D 100, 096004 (2019)

78. S. Laporta, Phys. Lett. B 772, 232 (2017)

79. R.H. Parker, C. Yu, W. Zhong, B. Estey, H. Müller, Science 360, 191 (2018)

80. A. Keshavarzi, D. Nomura, Th Teubner, Phys. Rev. D 101, 014029 (2020)

81. M. Davier, A. Hoecker, B. Malaescu, Z. Zhang, Eur. Phys. J. C 80, 241 (2020)
82. M. Benayoun, L. Delbuono, and F. Jegerlehner, Eur. Phys. J. C 80, 81 (2020); Eur. Phys. J. C 80, 244 (2020) (erratum)

83. Sz. Borsanyi, Z. Fodor, J.N. Guenther, C. Hoelbling, S.D. Katz, L. Lellouch, T. Lippert, K. Miura, L. Parato, K.K. Szabo, F. Stokes, B.C. Toth, Cs. Torok, and L. Varnhorst, eprint arXiv:2002.12347

84. K. Melnikov and A. Vainshtein, eprint arXiv:1911.05874

85. G. Colangelo, F. Hagelstein, M. Hoferichter, L. Laub, P. Stoffer, Phys. Rev. D 101, 051501 (2020)

86. Th Blum, N. Christ, M. Hayakawa, T. Izubuchi, L. Jin, C. Jung, C. Lehner, Phys. Rev. Lett. 124, 132002 (2020)

87. H. Lamm, Phys. Rev. A 95, 012505 (2017)

88. M.S. Fee, A.P. Mills Jr., S. Chu, E.D. Shaw, K. Danzmann, R.J. Chicherster, D.M. Zuckerman, Phys. Rev. Lett. 70, 1397 (1993)

89. K. Danzmann, M. Fee, S. Chu, Phys. Rev. A 39, 6072 (1989)

90. M.W. Ritter, P.O. Egan, V.W. Hughes, K.A. Woodle, Phys. Rev. A 30, 1331 (1984)

91. A.P. Mills Jr., G.H. Bearman, Phys. Rev. Lett. 34, 246 (1975)

92. A.P. Mills Jr., Phys. Rev. A 27, 262 (1983)

93. D. Eiras, J. Soto, Phys. Lett. 491B, 101 (2000)

94. V.G. Ivanov, E.Yu. Korzinin, S.G. Karshenboim, Phys. Rev. D 80, 027702 (2009)

95. A.P. Martynenko, Phys. Rev. A 71, 022506 (2005)

96. A.B. Mickelwait, H.C. Corben, Phys. Rev. 96, 1145 (1954)

97. G.E. Pustovalov, Sov. Phys. JETP 5, 1234 (1957)

98. D.D. Ivanenko, G.E. Pustovalov, Adv. Phys. Sci. 61, 1943 (1957)

99. S.G. Karshenboim, Can. J. Phys. 76, 169 (1998)

100. S.G. Karshenboim, JETP 89, 850 (1999)

101. S.G. Karshenboim, V.G. Ivanov, V.M. Shabaev, Can. J. Phys. 76, 503 (1998)

102. S.G. Karshenboim, V.G. Ivanov, V.M. Shabaev, JETP 90, 59 (2000) 